\title{
Las mujeres en alerta por violencia de género en espacios públicos. Comparativo con tres escuelas de educación superior públicas en México
}

\author{
Women on alert for gender violence in public spaces. Comparison of three \\ public higher education schools in Mexico \\ Mulheres em alerta para violência de gênero em espaços públicos. \\ Comparação com três escolas públicas de ensino superior no México
}

\author{
Claudia Alejandra Hernández Herrera \\ Instituto Politécnico Nacional, México \\ cahernandezh@ipn.mx \\ http://orcid.org/0000-0002-4060-2941
}

\section{Resumen}

El acoso sexual callejero es un tipo de violencia de género que pone en evidencia las desigualdades entre mujeres y hombres, y la dominación masculina en los espacios públicos. El escaso sentido de inseguridad, aunado a la insuficiente confianza en los procesos de denuncia y la impunidad, generan en las mujeres y en las niñas un miedo constante cuando deben transitar por espacios públicos, lo cual vulnera sus derechos de movilidad y bienestar. Por eso, el objetivo de este artículo fue estudiar las percepciones de las universitarias con respecto a las manifestaciones severas y a las conductas de acoso sexual que les generan miedo e inseguridad. De ese modo, se procuró identificar los escenarios de violencia de género a los que se enfrentan las estudiantes. Para ello, se diseñó un estudio transversal cuantitativo, con una muestra por conveniencia de 1656 mujeres estudiantes de tres escuelas públicas de enseñanza superior en México. Los resultados demuestran que las participantes perciben la presencia constante de acosadores y hostigadores sexuales en las universidades. Asimismo, se identificaron algunas situaciones habituales que viven las universitarias que han sido víctimas de acoso, lo cual las ha 


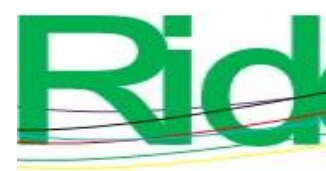

Revista Iberoamericana para la Investigación y el Desarrollo Educativo ISSN $2007-7467$

obligado a reforzar sus estrategias de evasión. Por tanto, se concluye que es necesaria la intervención urgente de los gobiernos y las universidades con acciones contundentes y con cuerpos policiales sensibles a la perspectiva de género.

Palabras claves: acosadores y hostigadores sexuales, acoso sexual, espacios públicos, mujeres, violencia de género.

\begin{abstract}
Street sexual harassment is a type of gender violence that highlights inequalities between women and men, and male domination in public spaces. The low sense of insecurity, coupled with insufficient trust in reporting processes and impunity, keeps women and girls in constant fear in public spaces, and blocks their right to mobility and well-being. The objective of this article is to study the perceptions of university students regarding severe manifestations and sexual harassment behaviors that generate fear and insecurity, in order to identify the scenarios of gender violence that female students face. It is a quantitative cross-sectional study with a convenience sample of 1,656 female students from three public higher education schools in Mexico. It was found that there is a constant identification of harassers and sexual harassers in universities. Some factors experienced by female university students who have been victims of severe behaviors that put them at risk were identified, as well as factors in common among young women that reinforce their avoidance strategies. It is concluded that it is necessary the intervention of governments and universities with affirmative actions, and with police forces sensitive to the gender perspective, within the framework of the declarations of alerts of gender violence against women.
\end{abstract}

Keywords: harassers and sexual harassers, sexual harassment, public spaces, women, gender violence. 


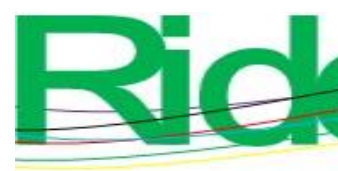

Revista Iberoamericana para la
Investigación y el Desarrollo Educativo ISSN 2007-7467

\section{Resumo}

O assédio sexual nas ruas é um tipo de violência de gênero que destaca as desigualdades entre mulheres e homens e a dominação masculina nos espaços públicos. O baixo sentimento de insegurança, somado à falta de confiança nos processos de denúncia e impunidade, geram nas mulheres e meninas um medo constante quando devem se deslocar nos espaços públicos, o que viola seus direitos à mobilidade e ao bem-estar. Portanto, o objetivo deste artigo foi estudar a percepção de universitários quanto às manifestações graves e comportamentos de assédio sexual que geram medo e insegurança. Dessa forma, buscou-se identificar os cenários de violência de gênero que os alunos enfrentam. Para tanto, foi elaborado um estudo transversal quantitativo, com uma amostra de conveniência de 1.656 alunas de três escolas públicas de ensino superior do México. Os resultados mostram que os participantes percebem a presença constante de assediadores e assediadores sexuais nas universidades. Da mesma forma, foram identificadas algumas situações comuns vividas por estudantes universitários vítimas de assédio, o que os obrigou a reforçar suas estratégias de evitação. Portanto, conclui-se que é necessária a intervenção urgente de governos e universidades com ações contundentes e com forças policiais sensíveis à perspectiva de gênero.

Palavras-chave: assediadores e assediadores sexuais, assédio sexual, espaços públicos, mulheres, violência de gênero.

Fecha Recepción: Marzo 2021

Fecha Aceptación: Septiembre 2021

\section{Introducción}

De acuerdo con el Fondo de Población de las Naciones Unidas, la violencia por razones de género es un tema que aún no ha recibido la atención requerida. De hecho, se sabe en la actualidad a muchas mujeres se les prohíbe trabajar o asistir a lugares públicos, donde en muchas ocasiones son víctimas de manifestaciones verbales con expreso contenido sexual (ONU Mujeres, 2020). En este sentido, en una considerable cantidad de literatura científica se afirma que la violencia contra las mujeres se encuentra presente en las ciudades, lo que genera desigualdad y discriminación (Hawken, Leao Gudes, Izadpanahi, Viswanath y Pettit, 2020). 


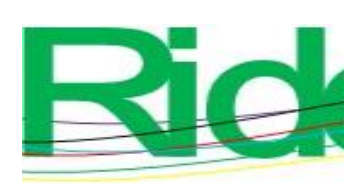

Revista Iberoamericana para la Investigación y el Desarrollo Educativo ISSN $2007-7467$

están más expuestas a sufrir acoso sexual, ya que se encuentran cautivas en el tránsito y no tienen acceso a medios de transporte particulares.

Por su parte, Hernández y Gómez (2021) encontraron en una universidad de Ciudad de México que algunos profesores acosan selectivamente a las alumnas, a las cuales les solicitan determinadas formas de vestir al momento de presentar exámenes. En tal sentido, investigaciones realizadas por Campbell et al. (2021) encontraron que uno de cada ocho estudiantes universitarios ha sido víctima de incidentes sexuales. Para ellas, por tanto, el acoso es un tema habitual al cual deben apartarse para lograr el objetivo de concluir su carrera.

Sin embargo, en otros casos las estudiantes se han organizado en movimientos de resistencia. Así han surgido iniciativas en redes sociales para evidenciar el acoso sexual y cómo este se encuentra ampliamente relacionado con el género. Un ejemplo es la iniciativa \#Miprimeracoso, que divulga las experiencias vividas por ellas, donde $62 \%$ de los agresores son hombres y $47 \%$ de los episodios se han producido en los espacios públicos.

En cuanto a iniciativas más formales, se han creado observatorios contra el acoso sexual callejero en países como Chile, Guatemala, Bolivia, Costa Rica, Uruguay, Nicaragua y Colombia, aunque aún falta atención y profundidad en este tema debido a la gravedad de las consecuencias que genera (López, 2020). Por ejemplo, cuando se habla de planificación urbana, debe quedar claro que las mujeres tienen otras necesidades al momento de trasladarse en transporte público, donde son más vulnerables a sufrir agresiones sexuales (Quinones, 2020).

De hecho, en el caso de la Ciudad de México y el Estado de México, se debe prever que cuentan con alertas por violencia de género contra las mujeres, y que el EDOMEX posee una doble alerta por la desaparición de mujeres, niñas y adolescentes, a lo cual se debe sumar las cifras de feminicidios y el aumento del enojo de las estudiantes por los casos de acoso y hostigamiento sexual que se presentan en las universidades.

Descrito el panorama anterior, en la presente investigación se planteó la siguiente interrogante: ¿cuáles son las apreciaciones de las mujeres de enseñanza superior en relación con la violencia de género, con énfasis en el acoso sexual y la seguridad en los espacios públicos? El objetivo, por tanto, fue estudiar las percepciones de las universitarias con respecto a los siguientes criterios: manifestaciones severas y conductas de acoso sexual que generan miedo e inseguridad, estrategias de evasión, percepción del trabajo de los policías, seguridad en las calles y el transporte, apreciación del trabajo de la escuela sobre las 


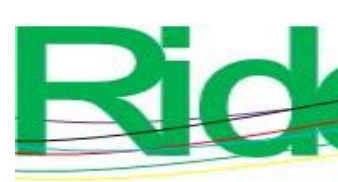

Revista Iberoamericana para la
Investigación y el Desarrollo Educativo
ISSN $2007-7467$

acciones de seguridad y abatimiento del acoso sexual, y apreciación sobre la existencia de acosadores y hostigadores en las escuelas. Lo anterior con el fin de identificar los posibles escenarios de violencia de género a los que se enfrentan.

\section{Marco teórico}

Las mujeres y las niñas del todo el mundo son víctimas constantes de acoso sexual y de otras manifestaciones de violencia en los espacios públicos (Ceccato y Laokaitou, 2021). Esto es particularmente más habitual en sociedades en donde prevalece el machismo, pues este no solo justifica la violencia y la interpreta como un castigo merecido, sino que también promueve la complicidad familiar y social, así como la impunidad de las autoridades (Castañeda, 2019).

La literatura señala que las restricciones de movilidad están relacionadas con el acoso sexual, que es consecuencia de las expresiones espaciales del patriarcado que limitan las oportunidades educativas y laborales (Hebert et al., 2020; Pedersen, 2020). De acuerdo con Condon et al. (2007), el miedo limita la libertad de las mujeres para moverse en la ciudad. El temor a la victimización sexual que pueden sentir las mujeres puede ser un obstáculo para motivar su participación en muchas esferas de la vida, lo que coarta sus derechos humanos (Chant y McIlwaine, 2016; ONU Mujeres, 2017).

Las investigaciones demuestran que el acoso sexual callejero es violencia de género y es una forma de discriminación que atenta contra la dignidad humana al hacer que se sientan vulnerables e inseguras en los espacios públicos. Este se caracteriza por la atención sexual no deseada por parte de extraños en público y abarca una serie de comportamientos como abucheos, silbidos, miradas fijas, manoseos, comentarios verbales, conversaciones prolongadas no deseadas, insinuaciones sexuales, entre otros, que en algunos casos pueden llegar a la agresión sexual y a la violación (Ahmad, Ahmad y Masood, 2020; Logan, 2015; Wesselmann y Kelly, 2010).

Ceccato y Laokaitou (2021) añaden que el acoso sexual se refiere a múltiples conductas lascivas que también pueden incluir el acecho, el exhibicionismo, el mostrar imágenes sexuales, las burlas, etc. Por su parte, López (2020) menciona que el acoso sexual callejero genera ambientes hostiles con consecuencias para la persona que recibe las insinuaciones o los ataques. 


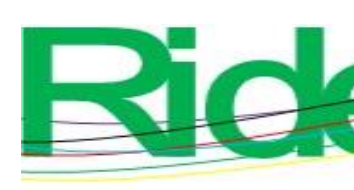

Revista Iberoamericana para la Investigación y el Desarrollo Educativo ISSN $2007-7467$

El acoso sexual callejero, en síntesis, se asocia con un conjunto de manifestaciones con connotación sexual que llevan implícitas relaciones de poder desiguales entre mujeres y hombres, las cuales lamentablemente han sido normalizadas y hasta justificadas en nuestra sociedad (Vallejo Rivera y Rivarola, 2013). Por ello, se puede afirmar que los espacios públicos son habitados de distinta manera por hombres y por mujeres, pues en estos ellas se encuentran en una posición de objeto (Cereceda, Patiño, Huenún y Romero, 2021; López, 2020;). En pocas palabras, el acoso callejero puede ser considerado como una forma de control social por parte de los hombres para continuar siendo dueños del espacio público (De Backer, 2020).

No en vano existe una constante preocupación por estudiar el acoso sexual, ya que se reconoce que es un tipo de violencia sistemática contra las mujeres debido a su género, en donde intervienen una relación de poder que jerarquiza las relaciones entre mujeres y hombres (Toro y Ochoa, 2017).

Las principales motivaciones que llevan a los varones a tener comportamientos de acoso sexual se encuentran en que consideran a la mujer como un objeto sexual. Los hombres que han llevado a cabo ese tipo de manifestaciones demuestran altos niveles de sexismo hostil, orientación al dominio social y masculinidad autoasignada (Walton y Pedersen, 2021).

Estudios previos han hallado que existe una brecha de género en el transporte que afecta la movilidad de las mujeres (Maffi, Malgieri y Di Bartolo, 2015). Por lo anterior, de acuerdo con Medina y Zapala (2016), algunas de las actitudes o estrategias que desarrollan las mujeres que han sido víctimas de acoso sexual se enfocan en cambiar la forma de vestir, en ir siempre acompañadas por un hombre o, en los casos más extremos, en restringir su movilidad (Corazón, 2016; Infante-Vargas y Boyer, 2021).

Las investigaciones han demostrado que la seguridad de las mujeres en los espacios públicos está relacionada con manifestaciones de acoso sexual, violación, discriminación sexual, asesinatos, feminicidios y ataques con ácido (Sen, Kaur y Zabiliūtė, 2020). De hecho, la evidencia demuestra que los lugares donde hay mayor incidencia de eventos de acoso sexual son dominados por los hombres (Mohamed y Stanek, 2019). Por ende, se suele asegurar que los espacios públicos en realidad solo son aprovechados y disfrutados por los varones (Vallejo Rivera y Rivarola, 2013). 


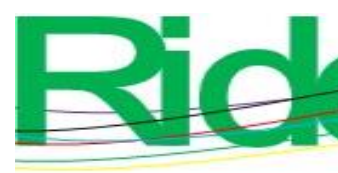

Revista Iberoamericana para la
Investigación y el Desarrollo Educativo
ISSN $2007-7467$

Para cambiar esta realidad se tiene que trasformar el entorno urbano y trabajar en la modificación de las conductas o predisposiciones delictivas (Davies, 2015). Una manera de detectar las variables que inciden en los delitos es identificar las formas en que las mujeres acceden a las ciudades. Además, se debe aplicar de forma amplia el concepto de ciudad inteligente para generar acciones desde la gobernanza con perspectiva de género (Bansal, Roychowdhury, Rawat, Narayan y Hoda, 2021), pues el diseño de la ciudad sigue reflejando la dominación masculina. Los gobiernos, por tanto, tienen la responsabilidad de contribuir con políticas públicas que incorporen la perspectiva de género, lo que significa comprender el sistema patriarcal que mantiene las relaciones de género que se reproducen históricamente a partir de la subordinación y desigualdad de la mujer (Hawken et al. 2020).

\section{Metodología}

El presente fue un estudio transversal cuantitativo. Los datos fueron recabados por medio de un cuestionario que se estructuró con base en la literatura científica. Se usó la escala tipo Likert con los siguientes valores: $1=$ totalmente en desacuerdo, 2 = en desacuerdo, $3=$ ni de acuerdo ni en desacuerdo, $4=$ de acuerdo, y $5=$ totalmente de acuerdo. En principio, se llevó a cabo un pilotaje para identificar el comportamiento del instrumento, el cual obtuvo resultados favorables con un alfa de Cronbach de 0.825.

La muestra fue por conveniencia y únicamente estuvo compuesta por mujeres. Se lograron aplicar 1656 instrumentos. A su vez, se llevó a cabo el análisis factorial exploratorio para identificar los factores (se obtuvieron siete), además de ejecutar la prueba estadística de Ómnibus para ubicar la existencia de los efectos de las variables en cada una de las escuelas con respecto a los factores encontrados (tabla 1). El comparativo se efectuó a través de la prueba de Rachas, que permite segmentar por escuela. Además, se trabajaron los modelos lineales generalizados que permiten examinar los efectos multivariables por medio de las pruebas de estadísticas de Ómnibus (tablas 3, 4 y 5). También se aplicó la prueba no paramétrica de Kruskal Wallis para determinar las diferencias entre los grupos integrados por cada una de las escuelas (tabla 6). Los datos se procesaron por medio del software SPSS. 
Tabla 1. Análisis factorial exploratorio

\begin{tabular}{|c|c|c|c|c|}
\hline $\begin{array}{l}\text { Factor } 1 \text {. Conductas severas de } \\
\text { acoso sexual }\end{array}$ & $\begin{array}{l}\text { Coeficiente } \\
\text { de } \\
\text { correlación }\end{array}$ & $\begin{array}{l}\text { Mujeres IPN } \\
\mathrm{n}=712 \\
\text { (promedios) }\end{array}$ & \begin{tabular}{|l|} 
Mujeres \\
TECNM \\
Chimalhuacán \\
$\mathrm{n}=442$ \\
(promedios) \\
\end{tabular} & $\begin{array}{l}\text { Mujeres TECNM } \\
\text { Gustavo A. } \\
\text { Madero } \mathrm{n}=502 \\
\text { (promedios) }\end{array}$ \\
\hline $\begin{array}{l}\text { En la calle o en el transporte } \\
\text { alguien ha intentado abusar } \\
\text { sexualmente de mí. }\end{array}$ & 0.781 & 1.46 & 1.43 & 1.37 \\
\hline $\begin{array}{l}\text { He sido víctima de un ataque } \\
\text { sexual por un desconocido. }\end{array}$ & 0.728 & 1.56 & 1.48 & 1.54 \\
\hline $\begin{array}{l}\text { Me han perseguido en la calle } \\
\text { con el propósito de acosarme } \\
\text { sexualmente. }\end{array}$ & 0.727 & 1.99 & 1.92 & 1.82 \\
\hline $\begin{array}{l}\text { Algún desconocido me ha } \\
\text { amenazado verbal o } \\
\text { físicamente. }\end{array}$ & 0.675 & 2.05 & 1.95 & 1.97 \\
\hline $\begin{array}{l}\text { Algún desconocido me ha } \\
\text { dado nalgadas que me han } \\
\text { hecho sentir mal. }\end{array}$ & 0.668 & 1.84 & 1.77 & 1.68 \\
\hline $\begin{array}{l}\text { Creo que es mejor quedarse } \\
\text { callada ante una situación en la } \\
\text { que alguien me está acosando } \\
\text { sexualmente, ya que considero } \\
\text { que está en peligro mi } \\
\text { integridad. }\end{array}$ & 0.644 & 1.7 & 1.65 & 1.54 \\
\hline $\begin{array}{l}\text { Alguien extraño me ha } \\
\text { mostrado sus genitales, en la } \\
\text { calle o en el transporte. }\end{array}$ & 0.637 & 2.14 & 2.18 & 2.12 \\
\hline $\begin{array}{l}\text { Algún desconocido se ha } \\
\text { masturbado frente de mí. }\end{array}$ & 0.422 & 2.16 & 2.12 & 1.91 \\
\hline $\begin{array}{l}\text { Factor } 2 . \text { Manifestaciones de } \\
\text { acoso sexual que generan } \\
\text { miedo e incomodidad }\end{array}$ & $\begin{array}{l}\text { Coeficiente } \\
\text { de } \\
\text { correlación }\end{array}$ & $\begin{array}{l}\text { Mujeres IPN } \\
\mathrm{n}=712 \\
\text { (promedios) }\end{array}$ & $\begin{array}{l}\text { Mujeres } \\
\text { TECNM } \\
\text { Chimalhuacán } \\
\mathrm{n}=442 \\
\text { (promedios) }\end{array}$ & $\begin{array}{l}\text { Mujeres TECNM } \\
\text { Gustavo A. } \\
\text { Madero } \mathrm{n}=502 \\
\text { (promedios) }\end{array}$ \\
\hline $\begin{array}{l}\text { Creo que algún extraño me ha } \\
\text { dicho palabras obscenas ya sea } \\
\text { en la calle o en el transporte. }\end{array}$ & 0.787 & 3.82 & 3.74 & 3.84 \\
\hline $\begin{array}{l}\text { Algún extraño me hizo sentir } \\
\text { incómoda con algún silbido. }\end{array}$ & 0.781 & 3.81 & 3.78 & 3.82 \\
\hline $\begin{array}{l}\text { Alguien me ha mirado de } \\
\text { forma obscena, lo que me ha } \\
\text { hecho sentir incomodidad, } \\
\text { incluso miedo. }\end{array}$ & 0.737 & 3.69 & 3.62 & 3.59 \\
\hline $\begin{array}{l}\text { En el transporte público, } \\
\text { alguien ha intentado estar tan } \\
\text { cerca de mí que me ha hecho }\end{array}$ & 0.726 & 3.48 & 3.28 & 3.10 \\
\hline
\end{tabular}




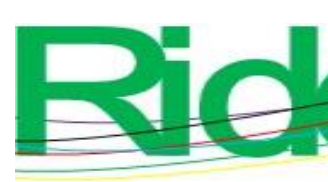

Revista Iberoamericana para la Investigación y el Desarrollo Educativo ISSN 2007 - 7467

\begin{tabular}{|c|c|c|c|c|}
\hline sentir incomodidad o miedo. & & & & \\
\hline $\begin{array}{l}\text { Cuando voy en el transporte o } \\
\text { estoy en la calle, alguien sin } \\
\text { mi consentimiento me ha } \\
\text { tomado fotografías. }\end{array}$ & 0.693 & 2.55 & 2.39 & 2.30 \\
\hline $\begin{array}{l}\text { En los últimos seis meses, } \\
\text { algún desconocido ha } \\
\text { intentado manosear mi cuerpo. }\end{array}$ & 0.627 & 2.31 & 2.22 & 2.00 \\
\hline $\begin{array}{l}\text { Algún desconocido me ha } \\
\text { tocado alguna parte del cuerpo } \\
\text { sin mi consentimiento. }\end{array}$ & 0.621 & 2.27 & 2.01 & 1.90 \\
\hline $\begin{array}{l}\text { Factor } 3 . \text { Estrategias de } \\
\text { evasión del acoso sexual }\end{array}$ & $\begin{array}{l}\text { Coeficiente } \\
\text { de } \\
\text { correlación }\end{array}$ & $\begin{array}{l}\text { Mujeres IPN } \\
\mathrm{n}=712 \\
\text { (promedios) }\end{array}$ & $\begin{array}{l}\text { Mujeres } \\
\text { TECNM } \\
\text { Chimalhuacán } \\
\mathrm{n}=442 \\
\text { (promedios) } \\
\end{array}$ & $\begin{array}{l}\text { Mujeres TECNM } \\
\text { Gustavo A. } \\
\text { Madero } \mathrm{n}=502 \\
\text { (promedios) }\end{array}$ \\
\hline $\begin{array}{l}\text { Considero que las mujeres } \\
\text { deben capacitarse en defensa } \\
\text { personal. }\end{array}$ & 0.709 & 4.29 & 4.3 & 4.29 \\
\hline $\begin{array}{l}\text { Creo que en los últimos seis } \\
\text { meses se ha incrementado la } \\
\text { inseguridad para las mujeres. }\end{array}$ & 0.694 & 4.19 & 4.07 & 3.98 \\
\hline $\begin{array}{l}\text { He tenido que cambiar mi } \\
\text { forma de vestir para evitar } \\
\text { acoso en la calle. }\end{array}$ & 0.679 & 3.16 & 3.21 & 3.13 \\
\hline $\begin{array}{l}\text { Creo que las mujeres no } \\
\text { debemos de salir a la calle } \\
\text { cuando obscurece, ya que hay } \\
\text { mucho peligro de sufrir } \\
\text { violencia. }\end{array}$ & 0.591 & 2.76 & 2.71 & 2.79 \\
\hline $\begin{array}{l}\text { He tenido que cambiar mis } \\
\text { rutas para evitar ser acosada. }\end{array}$ & 0.414 & 2.92 & 2.91 & 2.79 \\
\hline $\begin{array}{l}\text { Factor } 4 \text {. Percepción sobre el } \\
\text { trabajo de los policías }\end{array}$ & $\begin{array}{l}\text { Coeficiente } \\
\text { de } \\
\text { correlación }\end{array}$ & $\begin{array}{l}\text { Mujeres IPN } \\
\mathrm{n}=712 \\
\text { (promedios) }\end{array}$ & $\begin{array}{l}\text { Mujeres } \\
\text { TECNM } \\
\text { Chimalhuacán } \\
\mathrm{n}=442 \\
\text { (promedios) } \\
\end{array}$ & $\begin{array}{l}\text { Mujeres TECNM } \\
\text { Gustavo A. } \\
\text { Madero } \mathrm{n}=502 \\
\text { (promedios) }\end{array}$ \\
\hline $\begin{array}{l}\text { En caso de sufrir alguna } \\
\text { manifestación de acoso sexual, } \\
\text { me siento segura y confiada de } \\
\text { pedir ayuda a un policía. }\end{array}$ & 0.779 & 2.58 & 2.66 & 2.72 \\
\hline $\begin{array}{l}\text { Creo que la actuación de los } \\
\text { policías que resguardan la } \\
\text { seguridad de las mujeres es la } \\
\text { adecuada y están capacitados } \\
\text { para ello. }\end{array}$ & 0.777 & 2.32 & 2.26 & 2.46 \\
\hline
\end{tabular}




\begin{tabular}{|c|c|c|c|c|}
\hline $\begin{array}{l}\text { En caso de ser víctima de } \\
\text { violencia sexual en la calle, } \\
\text { estoy segura de que } \\
\text { denunciaría ante las } \\
\text { autoridades correspondientes. }\end{array}$ & 0.622 & 3.67 & 3.7 & 3.68 \\
\hline $\begin{array}{l}\text { Las personas se muestran } \\
\text { solidarias cuando observan o } \\
\text { detectan a alguien pidiendo } \\
\text { ayuda, y más si es mujer. }\end{array}$ & 0.591 & 2.94 & 2.92 & 2.89 \\
\hline $\begin{array}{l}\text { En caso de ser víctima de } \\
\text { acoso sexual en la calle, es } \\
\text { preferible pedir ayuda a una } \\
\text { policía mujer que a un policía } \\
\text { hombre. }\end{array}$ & 0.391 & 3.15 & 3.16 & 2.98 \\
\hline $\begin{array}{l}\text { Factor } 5 . \text { La seguridad en las } \\
\text { calles y el transporte }\end{array}$ & $\begin{array}{l}\text { Coeficiente } \\
\text { de } \\
\text { correlación }\end{array}$ & $\begin{array}{l}\text { Mujeres IPN } \\
\mathrm{n}=712 \\
\text { (promedios) }\end{array}$ & $\begin{array}{l}\text { Mujeres } \\
\text { TECNM } \\
\text { Chimalhuacán } \\
\mathrm{n}=442 \\
\text { (promedios) } \\
\end{array}$ & $\begin{array}{l}\text { Mujeres TECNM } \\
\text { Gustavo A. } \\
\text { Madero } \mathrm{n}=502 \\
\text { (promedios) }\end{array}$ \\
\hline $\begin{array}{l}\text { Las calles de la colonia en } \\
\text { donde vivo cuentan con } \\
\text { iluminación adecuada que me } \\
\text { hace sentir segura. }\end{array}$ & 0.817 & 2.66 & 2.56 & 2.59 \\
\hline $\begin{array}{l}\text { Creo que las calles de la } \\
\text { colonia son seguras para las } \\
\text { mujeres y las niñas. }\end{array}$ & 0.752 & 2.33 & 2.22 & 2.28 \\
\hline $\begin{array}{l}\text { El transporte público que } \\
\text { utilizo siento que es seguro. }\end{array}$ & 0.747 & 2.81 & 2.77 & 2.78 \\
\hline $\begin{array}{l}\text { Factor } 6 \text {. Apreciación del } \\
\text { trabajo de la escuela sobre las } \\
\text { acciones de seguridad y } \\
\text { abatimiento del acoso sexual }\end{array}$ & $\begin{array}{l}\text { Coeficiente } \\
\text { de } \\
\text { correlación }\end{array}$ & $\begin{array}{l}\text { Mujeres IPN } \\
\mathrm{n}=712 \\
\text { (promedios) }\end{array}$ & $\begin{array}{l}\text { Mujeres } \\
\text { TECNM } \\
\text { Chimalhuacán } \\
\mathrm{n}=442 \\
\text { (promedios) } \\
\end{array}$ & $\begin{array}{l}\text { Mujeres TECNM } \\
\text { Gustavo A. } \\
\text { Madero } \mathrm{n}=502 \\
\text { (promedios) } \\
\end{array}$ \\
\hline $\begin{array}{l}\text { En la escuela existen los } \\
\text { protocolos adecuados de } \\
\text { denuncia en caso de sufrir } \\
\text { acoso y hostigamiento sexual. }\end{array}$ & 0.712 & 3.31 & 3.23 & 3.54 \\
\hline $\begin{array}{l}\text { La escuela trabaja con } \\
\text { campañas de prevención de la } \\
\text { violencia de género. }\end{array}$ & 0.607 & 3.31 & 2.81 & 3.33 \\
\hline $\begin{array}{l}\text { Las sanciones que tienen la } \\
\text { escuela contra los acosadores } \\
\text { sexuales son ejemplares, por lo } \\
\text { que me siento segura si } \\
\text { denunció el acoso sexual. }\end{array}$ & 0.500 & 2.85 & 2.94 & 3.08 \\
\hline $\begin{array}{l}\text { En la escuela siento que estoy } \\
\text { en un espacio seguro. }\end{array}$ & 0.440 & 3.87 & 3.87 & 3.95 \\
\hline
\end{tabular}




\begin{tabular}{|l|r|r|l|l|}
\hline $\begin{array}{l}\text { Factor 7. Percepción sobre la } \\
\text { existencia de acosadores y } \\
\text { hostigadores en las escuelas }\end{array}$ & $\begin{array}{l}\text { Coeficiente } \\
\text { de } \\
\text { correlación }\end{array}$ & $\begin{array}{l}\text { Mujeres IPN } \\
\mathrm{n}=712 \\
\text { (promedios) }\end{array}$ & $\begin{array}{l}\text { Mujeres } \\
\text { TECNM } \\
\text { Chimalhuacán } \\
\mathrm{n}=442 \\
\text { (promedios) }\end{array}$ & $\begin{array}{l}\text { Mujeres TECNM } \\
\text { Gustavo A. } \\
\text { Madero n = 502 } \\
\text { (promedios) }\end{array}$ \\
\hline $\begin{array}{l}\text { Creo que en la escuela existen } \\
\text { alumnos que acosan } \\
\text { sexualmente a las compañeras. }\end{array}$ & 0.915 & 3.60 & 2.77 & \\
\hline $\begin{array}{l}\text { Creo que en la escuela existen } \\
\text { profesores que acosan a las } \\
\text { estudiantes. }\end{array}$ & 0.910 & 3.80 & & 2.91 \\
\hline
\end{tabular}

Fuente: Elaboración propia

\section{Resultados}

\section{Modelo lineal generalizado del factor uno. Manifestaciones severas de acoso sexual}

Este constructo examina las variables de acoso sexual severo (p. ej., ataque sexual, intento de abuso sexual, persecuciones, amenazas, mostrar genitales y masturbación en vía pública). En el caso del IPN se hallaron los efectos en las variables del tiempo de traslado $(\mathrm{p}=0.01<0.05)$, hora de llegada a casa $(\mathrm{p}=0.00<0.05)$, alcaldía $(\mathrm{p}=0.01<0.05)$, municipio del Estado de México $(\mathrm{p}=0.03<0.05)$, alguien acompaña a tomar el transporte $(\mathrm{p}=0.03<0.05)$, calificación a la seguridad del gobierno $(\mathrm{p}=0.001<0.05)$, escolaridad de la madre $(\mathrm{p}=0.01<0.05)$, ocupación de la madre $(\mathrm{p}=0.00<0.05)$, escolaridad del padre $(\mathrm{p}=0.01<0.05)$ y calificación a la escuela $(\mathrm{p}=0.00<0.05)($ tabla 2$)$.

Se encontró que las estudiantes del IPN que indicaron que tardan en trasladarse a la escuela de una hora a una hora y media son las que puntearon más alto las manifestaciones graves de acoso sexual. Además, las jóvenes que indicaron que llegan a casa entre las 23:00 y las 23:30 horas son las que han sido víctimas de expresiones severas de tipo sexual. Las estudiantes que calificaron alto los comportamientos nocivos de acoso sexual en espacios públicos son aquellas que habitan las alcaldías de a) Cuajimalpa, b) Coyoacán, c) Magdalena Contreras y d) Iztacalco. Por su parte, las jóvenes que provienen de los municipios de Tultitlán y Chimalhuacán son las que dieron más puntos a las acciones de acoso sexual.

Otro hallazgo fue que las mujeres que dijeron que no tenían quien las acompañara a tomar el transporte fueron las que calificaron más alto las manifestaciones. Un dato relevante que se encontró fue las que las mujeres que otorgan mayor cantidad de puntos a 


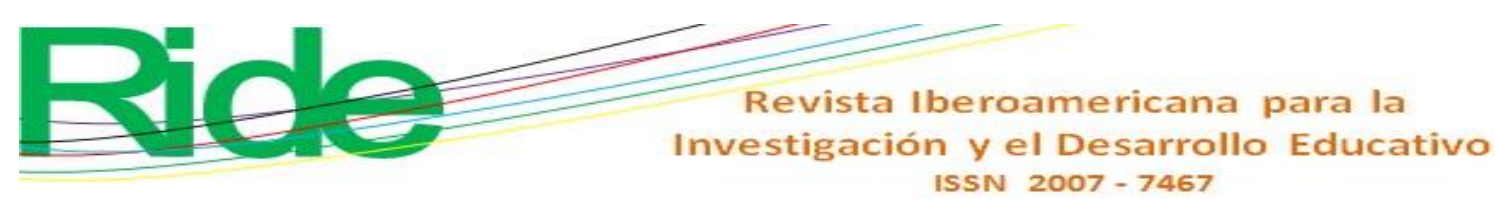

las manifestaciones graves son aquellas que consideran que la seguridad en sus alcaldías es excelente, lo que significa que es posible que la gravedad de los episodios de acoso se presente durante los trayectos. Se halló también que las estudiantes que dijeron que su mamá no tiene estudios o que cuenta con la enseñanza de primaria son las que otorgaron más puntos a las acciones de acoso sexual. Otro dato significativo fue que las estudiantes que han recibido más acciones de acoso sexual severo fueron aquellas cuyas madres estaban desempleadas. Por último, las mujeres que le dieron a la escuela una calificación de cinco (es decir, perciben como muy malo el actuar en materia de protección y seguridad hacia sus mujeres) fueron las que han estado mayormente expuestas a recibir manifestaciones de acoso sexual severo.

Las variables que obtuvieron efectos en el modelo del Tecnológico de Chimalhuacán se ubicaron en la afluencia de personas $(\mathrm{p}=0.00<0.05)$, seguridad en los trayectos $(\mathrm{p}=0.05=<0.05)$, calificación a la seguridad del gobierno $(\mathrm{p}=0.00<0.05)$, calificación a la escuela $(p=0.05=<0.05)$ y trabajo $(p=0.03<0.05)$ (tabla 2$)$. En el caso de las estudiantes del Tecnológico de Chimalhuacán se encontró que las jóvenes que perciben con mayor fuerza el acoso sexual severo son aquellas que consideran que las calles que recorren para llegar al transporte muestran algunas personas. Por otra parte, se halló que las estudiantes que califican altas las acciones de acoso son aquellas que califican como muy mala la seguridad de los trayectos. Además, se halló que las jóvenes que califican a la escuela como mala en materia de protección y seguridad son las que perciben con mayor intensidad las manifestaciones de acoso sexual en los espacios públicos. Por otro lado, las estudiantes que afirmaron estar trabajando son las que dieron más puntaje a las expresiones severas de acoso sexual en la calle.

Además, en el Tecnológico de Gustavo A. Madero se descubrieron los efectos en la variable alcaldía ( $p=0.00<0.05)$, seguridad en los trayectos $(p=0.00<0.05)$, ocupación de la madre $(\mathrm{p}=0.00<0.05)$, escolaridad del padre $(\mathrm{p}=0.01<0.05)$ y calificación a la escuela ( $p=0.03<0.05)$ (tabla 2). De igual forma se halló que las jóvenes del Tecnológico de Gustavo A. Madero que dieron mayor cantidad de puntos a las percepciones sobre las manifestaciones de acoso sexual severas son aquellas que expresaron que habitan en la delegación de Gustavo A. Madero e Iztacalco. También se encontraron variaciones en la puntuación de las expresiones agresivas de acoso sexual en aquellas que enfatizaron que la calle está sola cuando tienen que conseguir transporte público. Uno de los hallazgos interesante es que las estudiantes que califican como excelente la seguridad en los trayectos 

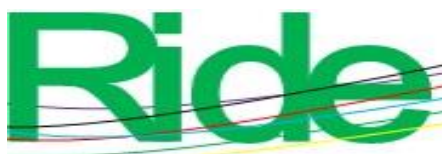

Revista Iberoamericana para la Investigación y el Desarrollo Educativo ISSN $2007-7467$

que realizan de la escuela a su casa son las que obtuvieron mayores puntos en relación con las acciones severas de acoso sexual, lo que podría indicar que cuando ellas efectúan otro tipo de movilidad es cuando pueden darse los episodios desagradables. Se encontró que las jóvenes que dijeron que sus madres trabajan en el gobierno y que sus padres tenían un posgrado son las que dieron mayor cantidad de puntos a las manifestaciones graves de acoso sexual. Por último, las estudiantes que otorgaron a la escuela una calificación mala en materia de protección y seguridad de sus estudiantes son las que lamentablemente han sido víctimas de manifestaciones de acoso sexual. 
Tabla 2. Modelos lineales generalizados, comparativo con las tres escuelas y los factores 1 , 2 у 3

\begin{tabular}{|c|c|c|c|c|c|c|c|c|c|}
\hline \multirow[t]{2}{*}{ Variables } & \multicolumn{3}{|c|}{$\begin{array}{c}\text { Factor 1. Conductas } \\
\text { severas de acoso sexual }\end{array}$} & \multicolumn{3}{|c|}{$\begin{array}{c}\text { Factor } 2 . \\
\text { Manifestaciones de } \\
\text { acoso sexual que } \\
\text { generan miedo e } \\
\text { incomodidad }\end{array}$} & \multicolumn{3}{|c|}{$\begin{array}{c}\text { Factor 3. Estrategias de } \\
\text { evasión del acoso } \\
\text { sexual }\end{array}$} \\
\hline & IPN & $\begin{array}{l}\text { TEC- } \\
\text { CHI }\end{array}$ & $\begin{array}{l}\text { TEC- } \\
\text { GAM }\end{array}$ & IPN & $\begin{array}{l}\text { TEC- } \\
\text { CHI }\end{array}$ & $\begin{array}{l}\text { TEC- } \\
\text { GAM }\end{array}$ & IPN & $\begin{array}{l}\text { TEC- } \\
\text { CHI }\end{array}$ & $\begin{array}{l}\text { TEC- } \\
\text { GAM }\end{array}$ \\
\hline $\begin{array}{l}\text { Tiempo de } \\
\text { traslado }\end{array}$ & $0.006^{*}$ & 0.473 & 0.858 & 0.251 & 0.640 & 0.824 & 0.070 & 0.408 & 0.830 \\
\hline $\begin{array}{l}\text { Hora de salida } \\
\text { de casa }\end{array}$ & 0.368 & 0.318 & 0.772 & 0.487 & $0.005^{*}$ & $0.043 *$ & 0.590 & 0.810 & 0.796 \\
\hline $\begin{array}{l}\text { Hora de } \\
\text { llegada a casa }\end{array}$ & $\begin{array}{r}0.000 * \\
*\end{array}$ & 0.757 & 0.204 & 0.327 & 0.214 & 0.245 & 0.067 & 0.256 & 0.805 \\
\hline Alcaldía & $0.01 *$ & 0.110 & $\begin{array}{r}0.002 \\
*\end{array}$ & $0.017 *$ & 0.523 & 0.065 & $0.048 *$ & 0.065 & 0.384 \\
\hline $\begin{array}{l}\text { Municipio del } \\
\text { EDOMEX }\end{array}$ & $0.031 *$ & 0.741 & 0.074 & 0.257 & 0.991 & $0.02 *$ & 0.120 & 0.237 & 0.065 \\
\hline $\begin{array}{l}\text { Afluencia de } \\
\text { personas }\end{array}$ & 0.100 & $0.005^{*}$ & $\begin{array}{r}0.016 \\
*\end{array}$ & 0.099 & $0.01 *$ & 0.179 & 0.308 & 0.331 & $0.01 *$ \\
\hline $\begin{array}{l}\text { Alguien te } \\
\text { acompaña a } \\
\text { tomar el } \\
\text { transporte }\end{array}$ & $0.003^{*}$ & 0.163 & 0.894 & 0.325 & $0.045^{*}$ & 0.928 & 0.950 & 0.414 & $0.01 *$ \\
\hline $\begin{array}{l}\text { Seguridad en } \\
\text { los trayectos }\end{array}$ & 0.528 & $0.051^{*}$ & $\begin{array}{r}0.002 \\
*\end{array}$ & $0.002 *$ & 0.499 & $0.037 *$ & 0.221 & 0.223 & $0.02 *$ \\
\hline $\begin{array}{l}\text { Calificación a } \\
\text { la seguridad/ } \\
\text { gobierno }\end{array}$ & $\begin{array}{r}0.001 * \\
*\end{array}$ & $\begin{array}{r}0.000 * \\
*\end{array}$ & 0.275 & $\begin{array}{r}0.000 * \\
*\end{array}$ & $\begin{array}{r}0.000 * \\
*\end{array}$ & $0.007 *$ & $\begin{array}{r}0.000 * \\
*\end{array}$ & $\begin{array}{r}0.001 * \\
*\end{array}$ & $\begin{array}{r}0.00 * \\
*\end{array}$ \\
\hline $\begin{array}{l}\text { Escolaridad } \\
\text { de la madre }\end{array}$ & $0.012 *$ & 0.659 & 0.518 & $0.04 *$ & 0.480 & 0.094 & 0.118 & $0.043^{*}$ & 0.442 \\
\hline $\begin{array}{l}\text { Ocupación de } \\
\text { la madre }\end{array}$ & $\begin{array}{r}0.002 * \\
* \\
\end{array}$ & 0.986 & $\begin{array}{r}0.003 \\
* \\
\end{array}$ & 0.221 & 0.673 & $0.02 *$ & 0.419 & 0.058 & 0.718 \\
\hline $\begin{array}{l}\text { Escolaridad } \\
\text { del padre }\end{array}$ & $0.008^{*}$ & 0.573 & $\begin{array}{r}0.007 \\
*\end{array}$ & 0.008 & 0.056 & 0.142 & 0.782 & 0.460 & $0.03 *$ \\
\hline $\begin{array}{l}\text { Ocupación del } \\
\text { padre }\end{array}$ & 0.987 & 0.205 & 0.310 & 0.199 & 0.230 & 0.874 & 0.572 & 0.952 & 0.102 \\
\hline $\begin{array}{l}\text { Calificación a } \\
\text { la escuela }\end{array}$ & $\begin{array}{r}0.001 * \\
*\end{array}$ & $0.05 *$ & $\begin{array}{r}0.034 \\
*\end{array}$ & $\begin{array}{r}0.000 * \\
*\end{array}$ & 0.291 & $\begin{array}{r}0.000 * \\
*\end{array}$ & $\begin{array}{r}0.001 * \\
*\end{array}$ & 0.521 & $0.05 *$ \\
\hline Trabajo & 0.092 & $0.035^{*}$ & 0.641 & $0.016^{*}$ & $\begin{array}{r}0.000^{*} \\
*\end{array}$ & 0.135 & 0.358 & 0.960 & 0.451 \\
\hline
\end{tabular}

Fuente: Elaboración propia $(* * \mathrm{p}<0.01, * \mathrm{p}<0.05)$ 


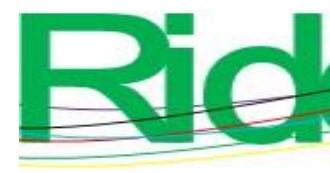

Revista Iberoamericana para la Investigación y el Desarrollo Educativo ISSN 2007 - 7467

Tabla 3. Modelos lineales generalizados, comparativo con las tres escuelas y los factores 4,5 y 6

\begin{tabular}{|c|c|c|c|c|c|c|c|c|c|}
\hline \multirow[t]{2}{*}{ Variables } & \multicolumn{3}{|c|}{$\begin{array}{l}\text { Factor } 4 \text {. Percepción sobre el } \\
\text { trabajo de los policías }\end{array}$} & \multicolumn{3}{|c|}{$\begin{array}{l}\text { Factor } 5 . \text { La seguridad en } \\
\text { las calles y el transporte }\end{array}$} & \multicolumn{3}{|c|}{$\begin{array}{c}\text { Factor 6. Apreciación del } \\
\text { trabajo de la escuela sobre } \\
\text { las acciones de seguridad y } \\
\text { abatimiento del acoso } \\
\text { sexual }\end{array}$} \\
\hline & IPN & $\begin{array}{l}\text { TEC- } \\
\text { CHI }\end{array}$ & $\begin{array}{l}\text { TEC- } \\
\text { GAM }\end{array}$ & IPN & $\begin{array}{l}\text { TEC- } \\
\text { CHI }\end{array}$ & $\begin{array}{l}\text { TEC- } \\
\text { GAM }\end{array}$ & IPN & $\begin{array}{l}\text { TEC- } \\
\text { CHI }\end{array}$ & $\begin{array}{l}\text { TEC- } \\
\text { GAM }\end{array}$ \\
\hline Tiempo de traslado & $0.006^{*}$ & 0.691 & 0.080 & 0.600 & 0.246 & 0.724 & 0.765 & 0,097 & 0.416 \\
\hline Hora de salida de casa & 0.323 & $0.034^{*}$ & 0.985 & 0.399 & 0.074 & $0.033^{*}$ & 0.111 & $0.030 *$ & 0.414 \\
\hline Hora de llegada a casa & $0.005^{*}$ & 0.140 & 0.249 & $0.009 *$ & 0.160 & 0.972 & $0.000 * *$ & 0.864 & $0.006^{*}$ \\
\hline Alcaldía & $0.022 *$ & 0.692 & $0.053 *$ & 0.413 & $0.041 *$ & 0.223 & $0.023^{*}$ & $0.030 *$ & 0.248 \\
\hline $\begin{array}{l}\text { Municipio del } \\
\text { EDOMEX }\end{array}$ & $0.001 *$ & 0.663 & 0.825 & $0.030 *$ & 0.053 & 0.691 & 0.120 & 0.956 & 0.155 \\
\hline Afluencia de personas & 0.232 & 0.763 & 0.485 & 0.070 & 0.446 & 0.055 & 0.829 & 0.994 & 0.108 \\
\hline $\begin{array}{l}\text { Alguien te acompaña a } \\
\text { tomar el transporte }\end{array}$ & 0.741 & 0.660 & 0.142 & 0.771 & 0.811 & 0.260 & 0.826 & 0.636 & 0.420 \\
\hline $\begin{array}{l}\text { Seguridad en los } \\
\text { trayectos }\end{array}$ & 0.721 & 0.645 & 0.411 & $0.000 * *$ & 0.000 & 0.192 & 0.212 & 0.301 & 0.757 \\
\hline $\begin{array}{l}\text { Calificación a la } \\
\text { seguridad/gobierno }\end{array}$ & $0.002 * *$ & 0.057 & $0.000 * *$ & $0.000 * *$ & 0.000 & $0.000 * *$ & 0.332 & $0.003 * *$ & $0.012 *$ \\
\hline $\begin{array}{l}\text { Escolaridad de la } \\
\text { madre }\end{array}$ & 0.129 & 0.072 & 0.333 & $0.042 *$ & 0.070 & 0.472 & 0.578 & 0.620 & 0.128 \\
\hline Ocupación de la madre & 0.198 & 0.375 & 0.249 & $0.003 *$ & 0.120 & 0.231 & $0.015^{*}$ & $0.045^{*}$ & 0.519 \\
\hline Escolaridad del padre & 0.129 & $0.001 * *$ & 0.220 & $0.008 *$ & $0.004 *$ & 0.279 & 0.076 & 0.620 & 0.289 \\
\hline Ocupación del padre & 0.197 & 0.390 & 0.621 & $0.003 *$ & 0.704 & 0.824 & 0.623 & 0.816 & 0.378 \\
\hline $\begin{array}{l}\text { Calificación a la } \\
\text { escuela }\end{array}$ & 0.034 & 0.398 & $0.035^{*}$ & 0.140 & 0.052 & 0.126 & $0.000^{* *}$ & $0.000^{* *}$ & $0.000^{* * *}$ \\
\hline Trabajo & 0.314 & 0.636 & 0.719 & 0.235 & 0.465 & 0.950 & 0.502 & 0.490 & 0.185 \\
\hline
\end{tabular}

Fuente: Elaboración propia $(* * p<0.01, * \mathrm{p}<0.05)$ 
Tabla 4. Modelos lineales generalizados, comparativo con las tres escuelas del factor 7

\begin{tabular}{|c|c|c|c|}
\hline \multirow{2}{*}{ Variables } & \multicolumn{3}{|c|}{$\begin{array}{c}\text { Factor } 7 . \text { Percepción sobre la } \\
\text { existencia de acosadores y } \\
\text { hostigadores en las escuelas }\end{array}$} \\
\hline & $\begin{array}{l}\text { IPN } \\
\text { Iztacalco }\end{array}$ & $\begin{array}{l}\text { TECNM } \\
\text { Chimalhuacán } \\
\end{array}$ & $\begin{array}{l}\text { TECNM } \\
\text { Gustavo } \\
\text { A. } \\
\text { Madero } \\
\end{array}$ \\
\hline Tiempo de traslado & 0.624 & 0.208 & 0.436 \\
\hline Hora de salida de casa & 0.059 & 0.564 & 0.259 \\
\hline Hora de llegada a casa & $0.016^{*}$ & 0.724 & 0.724 \\
\hline Alcaldía & 0.587 & 0.062 & 0.061 \\
\hline Municipio del EDOMEX & $0.023 *$ & 0.069 & 0.848 \\
\hline Afluencia de personas & $0.005 *$ & 0.206 & 0.291 \\
\hline $\begin{array}{l}\text { Alguien te acompaña a tomar el } \\
\text { transporte }\end{array}$ & 0.078 & 0.284 & 0.238 \\
\hline Seguridad en los trayectos & 0.420 & 0.003 & 0.091 \\
\hline $\begin{array}{l}\text { Calificación a la seguridad/ } \\
\text { gobierno }\end{array}$ & 0.532 & 0.238 & 0.117 \\
\hline Escolaridad de la madre & 0.658 & 0.323 & 0.981 \\
\hline Ocupación de la madre & 0.340 & 0.676 & 0.470 \\
\hline Escolaridad del padre & 0.653 & 0.072 & 0.327 \\
\hline Ocupación del padre & 0.162 & 0.793 & 0.583 \\
\hline Calificación a la escuela & $0.000 * *$ & $0.000 * *$ & $0.016 \cdots *$ \\
\hline Trabajo & 0.971 & 0.192 & 0.538 \\
\hline
\end{tabular}

Fuente: Elaboración propia $(* * p<0.01, * p<0.05)$ 


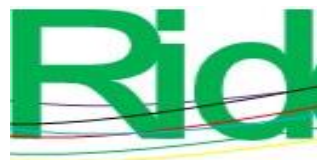

Tabla 5. Prueba de contraste de ómnibus y prueba estadística de Kruskal-Wallis, comparativo de los factores del 1 al 5

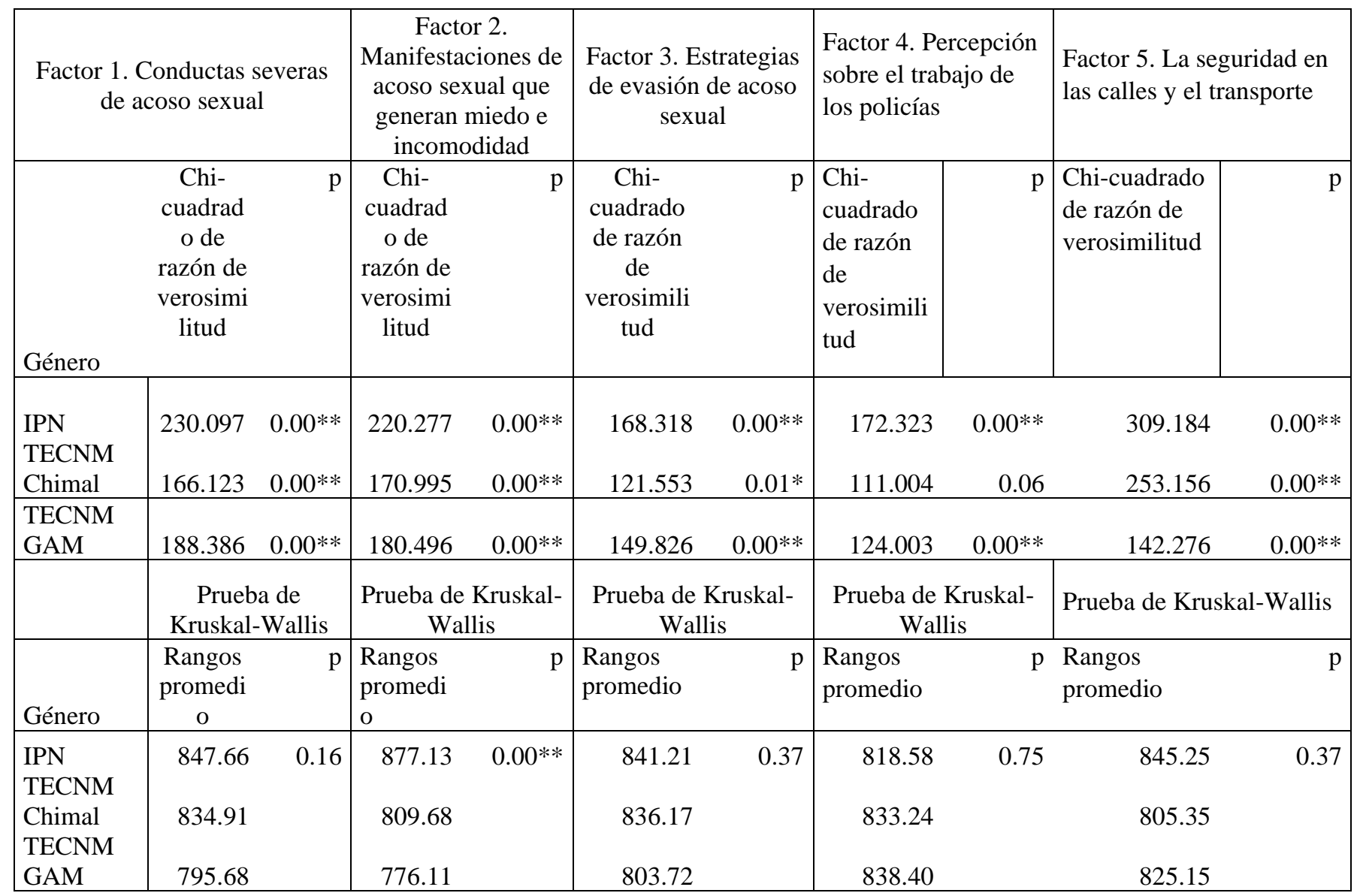

Fuente: Elaboración propia 


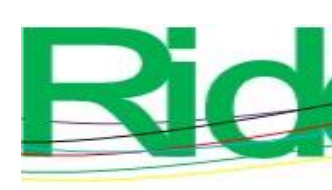

Revista Iberoamericana para la Investigación y el Desarrollo Educativo ISSN 2007 - 7467

Tabla 6. Prueba de contraste de ómnibus y prueba estadística de Kruskal-Wallis, comparativo de los factores 6 y 7

\begin{tabular}{|c|c|c|c|c|}
\hline \multicolumn{3}{|c|}{$\begin{array}{l}\text { Factor 6. Apreciación del trabajo de la escuela } \\
\text { sobre las acciones de seguridad y abatimiento } \\
\text { del acoso sexual }\end{array}$} & \multicolumn{2}{|c|}{$\begin{array}{l}\text { Factor } 7 . \text { Percepción sobre la } \\
\text { existencia de acosadores y } \\
\text { hostigadores en las escuelas }\end{array}$} \\
\hline Género & $\begin{array}{l}\text { Chi-cuadrado de } \\
\text { razón de } \\
\text { verosimilitud }\end{array}$ & $\mathrm{P}$ & $\begin{array}{l}\text { Chi-cuadrado de } \\
\text { razón de } \\
\text { verosimilitud }\end{array}$ & $\mathrm{P}$ \\
\hline IPN & 254.343 & $0.00 * *$ & 189.746 & $0.00 * *$ \\
\hline $\begin{array}{l}\text { TECNM } \\
\text { Chimal }\end{array}$ & 166.940 & $0.00 * *$ & 154.681 & $0.00 * *$ \\
\hline $\begin{array}{l}\text { TECNM } \\
\text { GAM }\end{array}$ & 194.566 & $0.00 * *$ & 130.270 & $0.00 * *$ \\
\hline & Prueba de Krus & allis & Prueba de Krus & \\
\hline Género & Rangos promedio & $\mathrm{P}$ & Rangos promedio & $\mathrm{P}$ \\
\hline $\begin{array}{l}\text { IPN } \\
\text { TECNM } \\
\text { Chimal } \\
\text { TECNM } \\
\text { GAM }\end{array}$ & $\begin{array}{l}824.96 \\
752.71 \\
900.25\end{array}$ & $0.00 * *$ & $\begin{array}{r}1035.71 \\
649.35 \\
692.44\end{array}$ & $0.00 * *$ \\
\hline
\end{tabular}

Fuente: Elaboración propia

\section{Modelo lineal generalizado del factor dos. Manifestaciones de acoso sexual que generan miedo e incomodidad}

En este factor se estudian las percepciones sobre conductas como silbidos, acercamientos indeseados, tomar fotografías, manoseos y tocamientos. En el caso del IPN, se hallaron los efectos en las variables de alcaldía $(\mathrm{p}=0.017<0.05)$, seguridad en los trayectos $(\mathrm{p}=0.002<0.05)$, calificación a la seguridad $(\mathrm{p}=0.000<0.05)$, escolaridad de la madre $(\mathrm{p}=0.04<0.05)$, calificación a la escuela en materia de seguridad $(\mathrm{p}=0.00<$ $0.05)$ y la estudiante trabaja $(\mathrm{p}=0.01<0.05)$ (tabla 2$)$. Las estudiantes que provienen de las alcaldías de Cuajimalpa, Magdalena Contreras y Gustavo A. Madero son las que han recibido mayor cantidad de acciones de acoso sexual que las incomodan; igual sucede con las jóvenes que perciben como mala la seguridad en los trayectos, las cuales han sido víctimas de esas conductas nocivas. 


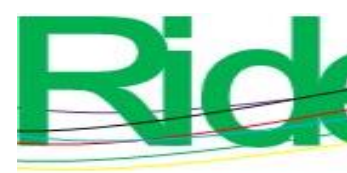

\section{Revista Iberoamericana para la Investigación y el Desarrollo Educativo ISSN 2007 - 7467}

Asimismo, las estudiantes que otorgan una calificación excelente al gobierno en materia de seguridad también han percibido acoso sexual por parte de extraños. Otro dato interesante es que las jóvenes que sienten más miedo por las agresiones de acoso sexual son las que señalaron que sus madres no tienen estudios o solo concluyeron la primaria. Por otra parte, las mujeres que percibieron con mayor fuerza las manifestaciones de acoso sexual son aquellas que le dieron una calificación de 5 (muy mala) a la escuela en materia de protección y seguridad. También se percibió que las estudiantes que trabajaban son las que califican más alto las conductas de acoso sexual que generan miedo.

Por su parte, en las jóvenes del Tecnológico de Chimalhuacán, los efectos del modelo se hallaron en la hora de salida de casa $(\mathrm{p}=0.00<0.05)$, afluencia de personas en las calles $(\mathrm{p}=0.01<0.05)$, alguien te acompaña a tomar el transporte $(\mathrm{p}=0.04<0.05)$, calificación que se le otorga al gobierno en materia de seguridad ( $\mathrm{p}=0.00<0.05)$ y si la alumna cuenta con trabajo $(\mathrm{p}=0.00<0.05)$ (tabla 2). Se halló que las mujeres que califican más alto las expresiones de acoso sexual son aquellas que señalaron que salen de casa muy temprano (entre las 4.30 a. m. y las 5:00 a. m.). De igual forma, se detectó que las estudiantes puntean más alto las expresiones de acoso sexual en lugares donde hay poca afluencia de personas. Aunado a lo anterior, se encontró que las estudiantes que indicaron que no tenían a alguien que las acompañara a tomar el transporte son quienes perciben con mayor frecuencia las manifestaciones de acoso sexual. Además, las mujeres que le dieron al gobierno una calificación negativa en materia de seguridad son las que han sido víctimas en mayor medida de esas expresiones de acoso en el espacio público. Por último, las mujeres que expresaron que trabajaban en el momento de realizar el estudio son las que en mayor medida han sufrido acoso sexual en las calles.

En relación con el modelo de las estudiantes del Tecnológico de Gustavo A. Madero, los efectos principales se hallaron en la hora de salida de la casa $(\mathrm{p}=004<0.05)$, municipio del Estado de México ( $\mathrm{p}=0.02<0.05$ ), calificación de la seguridad en los trayectos $(\mathrm{p}=0.03<0.05)$, calificación que le otorgan al gobierno en materia de seguridad $(\mathrm{p}=0.01<0.05)$, ocupación de la madre $(\mathrm{p}=0.02<0.05)$ y la calificación que le otorgan a la escuela en materia de protección y seguridad de las mujeres $(\mathrm{p}=0.00<0.05)$ (tabla 2$)$. Los hallazgos que se obtuvieron fueron que las mujeres que dieron más puntos a las manifestaciones de acoso sexual que provocan miedo son aquellas que salen de casa entre las 6:00 a. m. y las 7:00 a. m. A su vez, las estudiantes que han recibido con mayor 


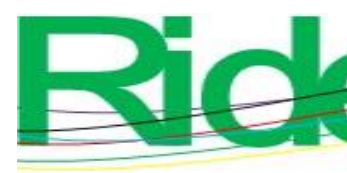

Revista Iberoamericana para la Investigación y el Desarrollo Educativo ISSN $2007-7467$

En el caso de las jóvenes del Instituto Tecnológico de Chimalhuacán, se encontraron variaciones en el modelo en la calificación que le otorgan al gobierno de su localidad en materia de seguridad $(\mathrm{p}=0.001<0.05)$ y la escolaridad de la madre $(\mathrm{p}=0.04<0.05)$ (tabla 2). Las jóvenes que calificaron al gobierno como malo en materia de seguridad son quienes han tenido que reforzar sus estrategias para evitar ser víctimas de acoso sexual. Además, se halló que las estudiantes que expresaron que sus madres tienen un posgrado son las que obtuvieron mayores puntos en materia de acciones para la prevención de acoso sexual.

Por último, en el caso de las jóvenes estudiantes del Tecnológico de Gustavo A. Madero, los efectos del modelo se ubicaron en la percepción sobre la afluencia de personas en la calle para conseguir transporte $(\mathrm{p}=0.01<0.05)$, alguien te acompaña a tomar el transporte $(\mathrm{p}=0.01<0.05)$, calificación de la seguridad en los trayectos $(\mathrm{p}=0.02<0.05)$, calificación que le otorgan al gobierno de su localidad en materia de seguridad ( $\mathrm{p}=0.00<$ $0.05)$, la escolaridad del padre $(\mathrm{p}=0.03<0.05)$ y la calificación que le otorgan a la escuela en materia de protección hacia sus mujeres (tabla 2). Se encontró que las jóvenes que han reforzado las acciones para intentar no ser víctimas del acoso sexual son aquellas que indicaron que la calle está sola en el momento que salen a conseguir algún medio de transporte. Además, se obtuvo un dato interesante, pues las jóvenes que han tenido que trabajar en sus estrategias para evadir el fenómeno son aquellas que cuentan con alguien que las acompaña a tomar el transporte, lo que indica que las manifestaciones se dan en los trayectos o en otros espacios. Por otra parte, las jóvenes que obtuvieron más puntos con respecto a las acciones para evitar el acoso son aquellas que consideran que la seguridad en los trayectos que realizan es mala. También las estudiantes que otorgaron una mala calificación en materia de seguridad al gobierno de su localidad son aquellas que han tenido que reforzar sus medidas de prevención. Otro hallazgo importante es que las mujeres que dijeron que sus padres tienen una licenciatura son las que han sido capaces de fortalecer sus estrategias de prevención contra el acoso sexual. Por último, se halló que las estudiantes que calificaron como mala la gestión en materia de protección y seguridad hacia las mujeres de parte de la escuela son el grupo de jóvenes que han fortalecido sus estrategias para esquivar el acoso sexual. 


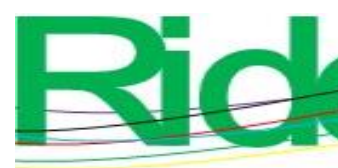

Revista Iberoamericana para la Investigación y el Desarrollo Educativo ISSN 2007-7467

\section{Modelo lineal generalizado del factor cuatro. Percepción sobre el trabajo de los policías}

Este factor analiza las variables relacionadas con la percepción sobre el rol que tienen los policías en los espacios públicos. Se cuestionó a las estudiantes sobre sus apreciaciones acerca de lo siguiente: a) si ellas llegaran a sufrir alguna manifestación de acoso sexual, ¿se sentirían seguras y confiadas de pedir ayuda a un policía?, b) creencias sobre la actuación de los policías para analizar si es la adecuada, ya que están capacitados para resguardar la seguridad de las mujeres, c) en caso de ser víctima de violencia sexual, ¿la alumna emitiría su denuncia ante las autoridades correspondientes?, d) ¿las personas se muestran solidarias al detectar que una mujer está en peligro o pide ayuda?, y e) en caso de ser víctima de acoso sexual, ¿es preferible pedir ayuda a un policía mujer?

En el caso de las jóvenes del IPN, se encontraron variaciones en el modelo en las variables de tiempo de traslado $(\mathrm{p}=0.006<0.05)$, hora de llegada a casa $(\mathrm{p}=0.005<$ 0,05), alcaldía $(\mathrm{p}=0.022<0.05)$, municipio del Estado de México $(\mathrm{p}=0.001<0.05)$ y calificación que le otorgan al gobierno en materia de seguridad ( $\mathrm{p}=0.002<0.05)$ (tabla 3). Se halló que las jóvenes que indicaron que realizan trayectos de más de dos horas son las que calificaron de mejor forma la percepción hacia el rol de los policías y la seguridad que les ofrecen en el tema del acoso sexual. Por su parte, las jóvenes que otorgaron más puntos al trabajo de los policías son aquellas que dijeron que llegaban a casa antes de las seis de la tarde, mientras que quienes menos puntos les otorgaron fueron las que llegaban entre las 11:30 y las 12 de la noche. Además, se encontró que las estudiantes que habitan en las alcaldías Xochimilco, Coyoacán y Venustiano Carranza son las que dieron más puntos a la percepción de seguridad que les ofrecen el trabajo de los policías en caso de presentarse alguna situación de acoso sexual; sin embargo, las que dieron menos puntos son las mujeres que habitan en las alcaldías de Milpa Alta y Cuajimalpa. De igual forma, se halló que las estudiantes que expresaron que habitan en los municipios de Chalco y Valle de Chalco son quienes otorgaron mayores puntos al sentido de seguridad que les brindan los policías en materia de acoso sexual, pero lo contrario sucede con los municipios de Naucalpan y Chimalhuacán. Además, las mujeres que calificaron como excelente la seguridad que ofrece el gobierno de su alcaldía son quienes puntearon más alto la apreciación que tienen de los policías; caso contrario sucede con las estudiantes que perciben de forma negativa el rol de los policías ante el tema de acoso sexual. 


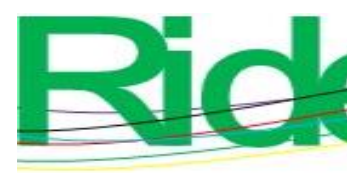

Revista Iberoamericana para la Investigación y el Desarrollo Educativo ISSN $2007-7467$

En cuanto a las mujeres que estudian en el Tecnológico de Gustavo A. Madero, las variaciones en el modelo se ubicaron en las variables de alcaldía $(\mathrm{p}=0.05<=0.05)$, calificación al gobierno de la localidad en materia de seguridad ( $\mathrm{p}=0.00<0.05$ ) y calificación a la escuela $(\mathrm{p}=0.03<0.05)$ (tabla 3). Las jóvenes que habitan en las alcaldías Cuauhtémoc, Iztacalco y Gustavo A. Madero son las que sienten mayor confianza hacia el trabajo de los policías en materia del tema de acoso sexual, y las que no perciben esa seguridad son las que habitan en Iztapalapa y Álvaro Obregón. Además, las jóvenes que perciben un poco más de seguridad (por lo que pueden pedir ayuda) son aquellas que califican como buena la seguridad que les ofrece el gobierno. Por último, las alumnas que califican de forma positiva el trabajo de la escuela en materia de protección y seguridad de las mujeres (por lo que se proclaman en contra del acoso sexual) son quienes manifestaron con mejores puntuaciones el trabajo de percepción del rol que tienen los policías en materia de acoso sexual.

\section{Modelo lineal generalizado factor cinco. La seguridad en las calles y el transporte}

Este factor estudia las apreciaciones de las mujeres en relación con la seguridad que perciben en las calles y en el transporte para ellas y las niñas. Los efectos principales en el modelo del IPN se encontraron en la hora de llegada a casa $(\mathrm{p}=0.000<0.05)$, municipio del Estado de México ( $\mathrm{p}=0.030<0.05)$, seguridad en los trayectos $(\mathrm{p}=0.000<0.05)$, calificación al gobierno local en materia de seguridad $(\mathrm{p}=0.00<0.05)$, escolaridad de la madre $(\mathrm{p}=0.04<0.05)$, ocupación de la madre $(\mathrm{p}=0.00<0.05)$, escolaridad del papa $(\mathrm{p}=$ $0.008<0.05)$ y ocupación del padre $(\mathrm{p}=0.00<0.05)$ (tabla 3). Se descubrió que las estudiantes que perciben de forma positiva la seguridad de las calles de su colonia y el transporte son aquellas que indicaron que llegan a casa entre las 9 y las 9:30 de la noche. Por otra parte, las jóvenes que sienten inseguridad en las calles y el transporte son las que habitan en Ecatepec y Tlalnepantla. Además, las jóvenes que calificaron como mala la seguridad en los trayectos son quienes sienten mayor inseguridad en los espacios públicos. También se halló que las jóvenes que califican como mala la seguridad que les brinda el gobierno de su localidad son quienes sienten mayor inseguridad en las calles y en el transporte. También se descubrió que las jóvenes que perciben con mayor énfasis la inseguridad en las calles son aquellas cuyas madres tienen la secundaria. De igual forma, se 


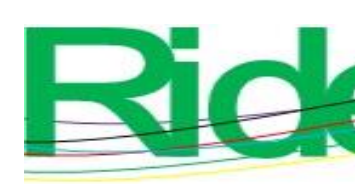

Revista Iberoamericana para la Investigación y el Desarrollo Educativo ISSN 2007-7467

encontró que las jóvenes que señalaron que sus padres no tienen estudios y que trabajan por su cuenta son las que sienten con mayor intensidad la inseguridad en los espacios públicos.

En el grupo de mujeres que se encuentran estudiando en el Tecnológico de Chimalhuacán, las variaciones se ubicaron en las variables de la alcaldía $(\mathrm{p}=0.04<0.05)$ y la escolaridad de los padres $(\mathrm{p}=0.00<0.05)$ (tabla 3). Se descubrió que las jóvenes que sienten en mayor medida la inseguridad son aquellas que expresaron que habitan en las delegaciones de Magdalena Contreras e Iztapalapa. De igual forma, se halló que las alumnas que declararon que sus padres no tenían estudios son las que perciben en mayor medida la inseguridad de las calles tanto para ellas como para las niñas.

En el caso del grupo de mujeres del Tecnológico de Gustavo A. Madero, las variaciones en el modelo se detectaron en la hora de salida $(\mathrm{p}=0.03<0.05)$ y calificación al gobierno de la localidad en materia de seguridad ( $\mathrm{p}=0.00<0.05)$ (tabla 3). Se encontró que las alumnas que indicaron que su hora de salida se ubicaba entre las 6:30 y las 7:00 son las que perciben con mayor énfasis la inseguridad en las calles. A su vez, las jóvenes que califican como mala la seguridad del gobierno de su localidad son quienes aprecian con mayor intensidad la inseguridad en las calles y en el transporte.

\section{Modelo lineal generalizado factor seis. Apreciación del trabajo de la escuela sobre las acciones de seguridad y abatimiento del acoso sexual}

Esta dimensión analiza los elementos que tienen relación con el trabajo de la escuela parar mantener un espacio seguro. Las variables que se estudiaron fueron las siguientes: precisar si las alumnas se sienten seguras en la escuela, identificar si en las escuelas existen los protocolos adecuados para hacer frente al acoso y al hostigamiento, determinar si la escuela trabaja con campañas de prevención de violencia de género, y señalar las sanciones de la escuela contra los acosadores y hostigadores sexuales.

En el caso del grupo de jóvenes del IPN, se encontraron los efectos en las variables de la hora de llegada a la casa $(\mathrm{p}=0.00<0.05)$, la alcaldía $(\mathrm{p}=0.02<0.05)$, ocupación de la madre $(p=0.01<0.05)$, calificación a la escuela en las acciones de seguridad, por lo que se proclama antiacoso $(\mathrm{p}=0.00<0.05)$ (tabla 3$)$. Las jóvenes que calificaron con baja puntuación el trabajo de la escuela en materia de espacio seguro son las que expresaron que llegan a casa entre las 11:30 y las 12 de la noche. Además, las estudiantes que dieron menos puntos a la escuela en materia de seguridad son las que dijeron que habitan en la 


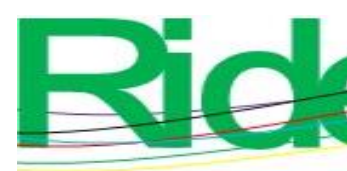

Revista Iberoamericana para la Investigación y el Desarrollo Educativo ISSN 2007 - 7467

alcaldía Milpa Alta y Miguel Hidalgo. También las alumnas que puntearon bajo el trabajo de la escuela son aquellas cuyas madres se dedican al hogar. Por último, se halló que las estudiantes que califican con baja puntuación el trabajo de la escuela son aquellas que consideran como malas las acciones implementadas contra el acoso, por lo que no se sienten en espacios seguros.

Por su parte, en las estudiantes del Tecnológico de Chimalhuacán se ubicaron las variaciones del modelo en la hora de salida de casa $(\mathrm{p}=0.03<0.05)$, la alcaldía $(\mathrm{p}=0.03<$ 0.05), la calificación que brinda el gobierno de la localidad en materia de seguridad ( $\mathrm{p}=$ $0.00<0.05)$, la ocupación de la madre $(\mathrm{p}=0.04<0.05)$ y la calificación que le otorgan a la escuela como espacio seguro $(\mathrm{p}=0.00<0.05)$ (tabla 3$)$. Las jóvenes que calificaron que perciben como inadecuado el trabajo de la escuela en relación con el tema de la seguridad hacia las jóvenes son las que declararon que salen de casa entre las 5:30 y las 6:00 de la mañana. Lo mismo de detectó en aquellas estudiantes que expresaron que habitan en la alcaldía de Álvaro Obregón. Sin embargo, se halló que las jóvenes que consideran como excelente el trabajo del gobierno de su localidad en el tema de seguridad son quienes otorgaron menos puntos al trabajo de la escuela. Además, se halló que las jóvenes cuyas madres estaban desempleadas son quienes puntearon más bajo el trabajo de la universidad en relación con el fenómeno de la seguridad. También se encontró que las participantes que califican con bajas puntuaciones el trabajo de la escuela son las que no logran percibir las estrategias implementadas.

En el caso de las mujeres del Tecnológico de Gustavo A. Madero, los efectos en el modelo se hallaron en la hora de llegada a casa $(\mathrm{p}=0.006<0.05)$, calificación que otorgan al gobierno de la localidad en materia de seguridad ( $\mathrm{p}=0.012<0.05)$ y calificación que le brindan a la escuela debido a que consideran que trabaja en el tema del acoso sexual ( $\mathrm{p}=$ $0.000<0.05$ ) (tabla 3). Se detectó que las jóvenes que brindaron menos puntos al trabajo de la escuela son quienes dijeron que llegan a casa entre las 11:00 y las 11:30 de la noche. Otro hallazgo interesante es que las mujeres que calificaron bajo el trabajo de la escuela son quienes otorgan mayores puntos al trabajo del gobierno de su localidad en materia de seguridad. También se halló que las jóvenes que califican como negativo el trabajo de la escuela son quienes no perciben iniciativas al respecto. 


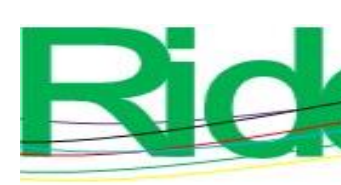

Revista Iberoamericana para la Investigación y el Desarrollo Educativo ISSN $2007-7467$

\section{Modelo lineal generalizado factor siete. Percepción sobre la existencia de acosadores y hostigadores en las escuelas}

En esta dimensión se evalúa la percepción sobre la existencia de acosadores y hostigadores en las escuelas. En las tres escuelas se hallaron efectos en la variable que mide la calificación que las estudiantes le brindan a la escuela en materia de seguridad hacia las mujeres, por lo que se proclaman escuelas en contra del acoso: IPN $(p=0.000<0.05)$, Tecnológico de Chimalhuacán ( $\mathrm{p}=0.000<0,05)$ y Tecnológico de Gustavo A. Madero ( $\mathrm{p}$ $=0.01<0.05$ ) (tabla 4). Se halló que las jóvenes que dieron calificación baja a las tres escuelas en materia de seguridad hacia las mujeres son las que perciben con mayor intensidad la presencia de acosadores y hostigadores en el lugar.

\section{Discusión}

En este trabajo se obtuvieron hallazgos importantes para poder entender el tema del acoso sexual en los espacios públicos. En el factor 1, que mide las manifestaciones graves de acoso sexual (abuso sexual, ataque sexual, persecuciones, mostrar genitales y masturbación), se encontró que las alumnas de las tres escuelas que han sido víctimas son aquellas que llegan tarde a casa, no hay alguien que las acompañe a tomar el transporte, expresan inseguridad en los trayectos, señalan que la calle está sola, trabajan y califican como negativas la seguridad y la protección de la escuela en materia de acoso y hostigamiento sexual. Además, señalan que sus madres no cuentan con estudios.

En el caso del factor que analiza las conductas de acoso que generan miedo e incomodidad (silbidos, palabras y miradas obscenas, acercamientos con malas intenciones y manoseos), se descubrió que las jóvenes que apreciaron como frecuentes esas acciones son aquellas que salen de su casa entre las 4:30 y las 5:00 de la mañana. Además, son jóvenes que se encuentran activas en el mercado laboral, no tienen quien las acompañe a tomar algún transporte seguro, perciben escasa afluencia de personas en la calle y señalan que es precaria la seguridad en los trayectos. En concreto, las estudiantes con mayores puntuaciones en este tipo de acciones nocivas son aquellas que viven en Ecatepec, Chalco y Valle de Chalco. Por último, las estudiantes del IPN y del TECNM son las que dieron más puntos a los comportamientos de acoso sexual incómodos son aquellas que califican como malo el trabajo de la escuela en materia de protección y seguridad. 


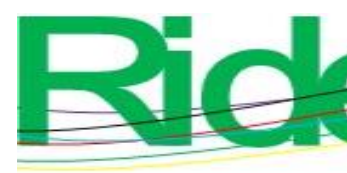

Revista Iberoamericana para la
Investigación y el Desarrollo Educativo
ISSN $2007-7467$

Los hallazgos encontrados aportan elementos a lo expuesto por Lewis, Saukko y Lumsden (2021), quienes encontraron que la mayor parte de las experiencias de acoso sexual en el transporte público tienen las siguientes características: a) depende de las horas en las que se viaja, b) las mujeres con frecuencia no responden al acoso sexual y c) este tipo de agresiones apenas es denunciada. De igual forma, las alumnas dieron mayores puntos a las agresiones sexuales cuando la calle está sola o presenta poca afluencia. Al respecto, Mohamed y Stanek (2019) encontraron que las calles muy transitadas brindan un sentido de seguridad por la vigilancia informal, pero en muchas ocasiones el número de personas ajenas a los barrios superan al número de residentes, por lo que las jóvenes deben mantenerse alerta. Otro hallazgo importante se encontró en las manifestaciones de acoso sexual, las cuales se presentan con mayor frecuencia en jóvenes que laboran. De acuerdo con Castañeda (2019), esto sucede porque las mujeres que trabajan y estudian deben trasladarse más frecuentemente de un lugar a otro, por lo que pasan más tiempo en los espacios públicos. En tal sentido, Vera-Gray (2016) añade que los marcadores sociales sitúan a las mujeres en una situación jerárquica, lo que genera desigualdad de género. Lamentablemente, el estudio teórico de la violación y el acoso sexual en espacios públicos cuenta con discursos que permiten justificar a los acosadores (Sadler, 2019). En consecuencia, el escenario no parece mejorar en relación con la inseguridad en las calles, por lo que ninguna mujer queda libre de ser víctima de feminicidio o desaparición (Guerrera, 2018).

En el factor tres (que analiza las estrategias de evasión del acoso sexual; p ej., tomar cursos de defensa personal, cambios en la forma de vestir, no salir cuando oscurece y cambios de rutas), se encontró que las jóvenes del IPN que han incrementado sus estrategias de evasión y sus creencias en torno al aumento de la inseguridad contra las mujeres son aquellas que percibieron como mala la seguridad que brinda la alcaldía donde habitan. En el caso de las alumnas del TECNM Chimalhuacán que han reforzado las acciones contra el acoso sexual, son aquellas que califican como mala la seguridad por parte del gobierno. Las mujeres del TECNM Gustavo A. Madero que han reforzado medidas son las que cuando salen de casa perciben que la calle está sola. Por último, las estudiantes del IPN y del TECNM de Gustavo A. Madero que han incrementado sus acciones para evitar el acoso son aquellas que perciben como mala la seguridad y protección de la escuela. 


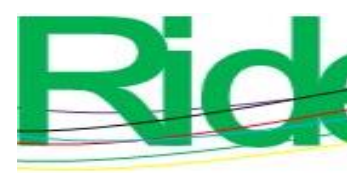

Revista Iberoamericana para la Investigación y el Desarrollo Educativo ISSN 2007 - 7467

Estos resultados concuerdan con los encontrados por Quinones (2020), López (2020) y Condon et al. (2017), quienes hallaron que las mujeres intentan protegerse de las manifestaciones de acoso sexual de la calle a través de cambios de ropa, tomar otras rutas sin importar que sean largas, no abordar el transporte si va lleno e incluso portar armas no letales para defenderse. La violencia que se presenta en las ciudades les exige a las mujeres tomar decisiones todo el día para evitar ser víctimas del acoso. Asimismo, Fleetwood (2019) explica que otras estrategias empleadas por las mujeres tienen que ver con pedir ayuda a otros y alejarse de los lugares donde se presentan esos episodios (Talone y Araujo, 2019).

En la dimensión cuatro (percepción sobre el trabajo de los policías), se obtuvo que las jóvenes del IPN que califican como mala esa labor son quienes llegan a casa entre las 11:30 y las 12 de la noche. Además, se enfatiza esta mala percepción del trabajo policial en aquellas jóvenes que habitan en Naucalpan y Chimalhuacán. Por último, las jóvenes del TECNM de Gustavo A. Madero que perciben seguridad por parte de los cuerpos policiales son aquellas que habitan en las alcaldías Iztapalapa y Álvaro Obregón.

Es lógico que las mujeres necesiten sentir seguridad y confianza hacia los cuerpos policiales para denunciar cualquier agresión sexual o delitos que pongan en riego su integridad. Sin embargo, como lo señalan Chubin (2014), Mellgren, Andersson e Ivert (2018), Dimitriou (2021), Ahmed, Yousaf y Asif (2019) y el Colectivo las tesis (2021), las víctimas de acoso sexual en muchas ocasiones se quedan calladas por la cultura de la vergüenza, lo que provoca que muchas sigan recibiendo una diversidad de agresiones en los espacios públicos. Como consecuencia, los agresores perciben que no existirá sanción y entonces seguirán actuando impunemente.

Otra de las razones por las que las mujeres no denuncian el acoso sexual es porque temen que esta sociedad patriarcal las culpe, lo que podría restringir aún más su movilidad. Por lo anterior, el movimiento feminista exige que se cuente con algún organismo que pueda brindar atención especializada para las víctimas de la violencia, ya que es evidente que este fenómeno se ha recrudecido, lo cual se evidencia en el incremento en los feminicidios (Torres Falcón, 2020).

En el factor cinco (que mide la percepción con respecto a la seguridad de las calles y el transporte), se evidenció que las jóvenes del IPN que expresaron mayor sentimiento de inseguridad en las calles son aquellas que viven en Ecatepec y Tlalnepantla. Estas, además, 


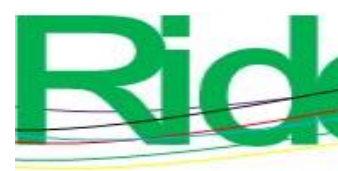

Revista Iberoamericana para la Investigación y el Desarrollo Educativo ISSN 2007 - 7467

califican como mala la seguridad en los trayectos. Por su parte, las estudiantes del TECNM Chimalhuacán que sienten mayor inseguridad son las que habitan en Magdalena Contreras e Iztapalapa. Un hallazgo interesante es que las participantes del IPN y del TECNM Chimalhuacán que perciben mayor inseguridad son aquellos que señalaron que sus padres no tenían estudios. Por último, las mujeres del TECNM Gustavo A. Madero que sienten mayor inseguridad son aquellas que salen de casa entre las 6:30 y las 7:00 de la mañana. Además, califican como malo el trabajo del gobierno.

Platzer (2016) expresa que es necesario la construcción de ciudades seguras para que las personas no tengan miedo de ser agredidas o violentadas. Sin embargo, para lograr esto se deben tomar en cuenta las percepciones de los afectados. Además, estamos de acuerdo con Lacey, Miller, Reeves y Tankel (2021) cuando expresan que se debe trabajar con urgencia en el tema de la seguridad en las calles a partir de enfoques de interseccionalidad, ya que permiten abordar el género como parte de una serie compleja de divisiones sociales, identidades y estructuras que dan forma a las experiencias singulares y colectivas de los individuos.

De igual manera, se coincide con lo expuesto por Barnes (2021) sobre la participación activa de las mujeres en la elaboración de las medidas de seguridad. En tal sentido, se sugiere la implementación de paneles de control de seguridad, plataformas digitales y mapas de riesgo (Hawken et al., 2013; Royo, Parikh y Belur, 2020).

Por último, con respecto al trabajo de la escuela en materia de seguridad y abatimiento del acoso sexual, se encontró que las estudiantes que otorgan bajas calificaciones en este criterio son las que perciben como malo el trabajo de las unidades académicas. Se tiene que poner cuidado en los resultados, ya que se encontró que las estudiantes de las tres instituciones señalaron la existencia de profesores acosadores (64\% del IPN, $30 \%$ del TECNM Gustavo A. Madero, y 27 \% del TECNM Chimalhuacán). Asimismo, otro de los elementos que impide que las jóvenes se sientan en espacios seguros dentro de las universidades es la presencia de alumnos que también las acosan (53\% del IPN, $29 \%$ del TECNM Gustavo A Madero, y 27 \% del TECNM Chimalhuacán). En pocas palabras, el acoso sexual en las universidades es un fenómeno común (pocas veces denunciado) debido a las limitaciones institucionales y a las creencias culturales de las víctimas (Dogar, 2021). 


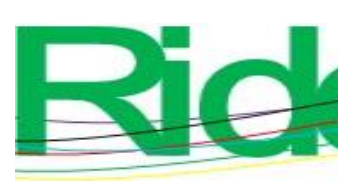

Revista Iberoamericana para la Investigación y el Desarrollo Educativo ISSN 2007-7467

\section{Conclusiones}

Las mujeres y las niñas salen a las calles con una serie de temores que muchas veces son inadvertidos por las autoridades (p. ej., ser agredidas, asaltadas, víctimas de ataques sexuales o asesinadas), de ahí que ellas tengan que adoptar medidas improvisadas para disminuir esos riesgos. Esta realidad, lógicamente, provoca que su tranquilidad emocional se vea alterada constantemente en cada trayecto que deban hacer hacia su lugar de estudio o trabajo.

Por eso, es imprescindible que exista un seguimiento de las estrategias implementadas para combatir este fenómeno de agresión hacia la mujer, así como exigir a los gobiernos la implementación de iniciativas que en otros países han permitido mejorar la seguridad pública. Además, se debe trabajar en acciones orientadas a la deconstrucción de las ideas machistas y androcéntricas.

En este trabajo quedan en evidencia las desigualdades que viven las mujeres y las niñas en los espacios públicos, por lo que se tienen que intensificar los esfuerzos para continuar con la construcción de senderos seguros, en especial en aquellas alcaldías y municipios considerados altamente violentos.

Asimismo, se tiene que animar a las mujeres a formar patrullajes ciudadanos para apoyar a aquellas jóvenes que llegan tarde a sus hogares, de modo que se pueda actuar de manera inmediata en caso de que se perciban factores de riesgo. Aunado a esto, se deben implementar medidas más severas en contra de los acosadores, hostigadores y feminicidas, pues en este trabajo se hace evidente que las mujeres y las niñas son grupos altamente vulnerables que necesitan protección por parte de la sociedad.

Por último, el miedo no se combate con victimismo, sino con acciones, por lo que se requiere que las universidades y los gobiernos trabajen y difundan las alertas de violencia de género. Además, las escuelas deben trabajar con la comunidad en temas relacionados con estereotipos, violencia generada a partir de las ideas del amor romántico, prevención del delito, creación de la policía de género y, en general, crear una red universitaria contra la violencia de género.

Finalmente, para futuros trabajos se pueden abordar los temas relacionados con la violencia en el noviazgo, así como indagar en el sentir de la comunidad universitaria sobre el tema de los tendederos que permiten evidenciar los actos de violencia de género. Por último, se puede entrevistar a estudiantes activas en los grupos de lucha feministas para 


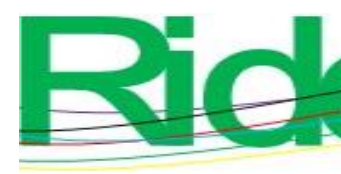

Revista Iberoamericana para la Investigación y el Desarrollo Educativo ISSN $2007-7467$

indagar sobre sus apreciaciones en torno al actuar de las autoridades frente al acoso y al hostigamiento sexual.

\section{Referencias}

Ahmad, N. M., Ahmad, M. M. and Masood, R. (2020). Socio-psychological Implications of Public Harassment for Women in the Capital City of Islamabad. Indian Journal of Gender Studies, 27(1), 77-100. Doi: https://doi.org/10.1177/0971521519891480.

Ahmed, B., Yousaf, F. N. and Asif, U. E. R. (2019). Combating Street Harassment: A Challenge for Pakistan. Women \& Criminal Justice, 31(4), 1-11. Doi: https://doi.org/10.1080/08974454.2019.1644697.

Álvarez León, V., Barrón Jiménez, D., Mejía Salgado, F. y Ortega Chávez, M. A. (2019). Percepción de la inseguridad frente al acoso sexual en la Ciudad de México. Repositorio Institucional REIMS (Red de Universidades La Salle). Memorias del Concurso Lasallista de Investigación, Desarrollo e Innovación.

Bansal, T., Roychowdhury, P., Rawat, P. Narayan, A. and Hoda, N. (2021). Gender and smart city: canvassing (in)security in Delhi. GeoJournal, 1-19. Doi: https://doi.org/10.1007/s10708-020-10369-z.

Barnes J. L. (2021). Designing Resilient Cities that Work for Women, Too. In Karácsonyi D., Taylor A. and Bird D. (eds), The Demography of Disasters. Springer, Cham. Doi: https://doi.org/10.1007/978-3-030-49920-4_9.

Campbell, J. C., Sabri, B., Budhathoki, C., Kaufman, M. R., Alhusen, J. and Decker, M. R. (2021). Unwanted sexual acts among university students: Correlates of victimization and perpetration. Journal of Interpersonal Violence, 36(1-2), 504-526. Doi: https://doi.org/10.1177/0886260517734221.

Castañeda (2019). El machismo invisible. México: De Bolsillo.

Ceccato, V. and Loukaitou, A. (2021). Fear of sexual harassment and its impact on safety perceptions in transit environments: a global perspective. Violence Against Women, 28(1). Doi: https://doi.org/10.1177/1077801221992874.

Cereceda, F. G., Patiño, V. G., Huenún, C. H. y Romero, B. L. (2021). Factores socioculturales y acoso callejero hacia mujeres: una perspectiva de hombres estudiantes universitarios. Revista de Estudiantes de Terapia Ocupacional, 8(1), 90111. 


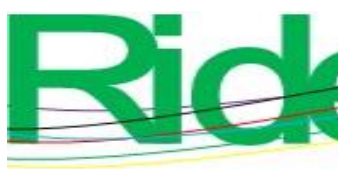
Revista Iberoamericana para la
Investigación y el Desarrollo Educativo
ISSN $2007-7467$

Chant, S. and McIlwaine, C. (2016). Cities, slums and gender in the global south: Towards a feminised urban future. Routledge.

Chubin, F. (2014). You may smother my voice, but you will hear my silence: An autoethnography on street sexual harassment, the discourse of shame and women's resistance in $\quad$ Iran. Sexualities, $\quad$ 17(1-2), 176-193. Doi: https://doi.org/10.1177/1363460713511097_.

Colectivo las tesis (2021). Quemar el miedo, un manifiesto. México: Planeta.

Condon, S., Lieber, M. and Maillochon, F. (2007). Feeling unsafe in public places: Understanding women's fears. Revue Française de Sociologie, 48(5), 101-128.

Corazón, R. (2016). Percepción y actitudes frente al acoso sexual callejero en estudiantes mujeres de una Universidad Privada de Medicina. Horizonte Médico, 16(1), 62-68.

Damián, A. and Flores, J. (2018). Feminicidios y políticas públicas: declaratorias de alertas de violencia de género en México, 2015-2017. Revista Perspectiva Geográfica, 23(2), 33-57.

Davies, W. (2015). Safe Cities and Communities. In Davies, W. (eds.), Theme Cities: Solutions for Urban Problems. GeoJournal Library (vol. 112). Springer, Dordrecht. Doi: https://doi.org/10.1007/978-94-017-9655-2_12.

De Backer, M. (2020). Street harassment and social control of young Muslim women in Brussels: destabilising the public/private binary. Journal of Gender-Based Violence. 4(3), 343-358. Doi: https://doi.org/10.1332/239868020X15986402157121.

Dimitriou, E. (2021). What is a reasonable response to sexual harassment? (thesis or dissertation). $\quad$ Retrieved from https://ore.exeter.ac.uk/repository/bitstream/handle/10871/124954/DimitriouE.pdf?s equence $=1 \&$ is Allowed $=\mathrm{y}$

Dogar, A. A. (2021). Perception, Prevalence and Awareness of Sexual Harassment among University Students in Abbottabad. Psychology and Education Journal,58(1), 1182-1195. Doi: https://doi.org/10.17762/pae.v58i1.869.

Fleetwood, J. (2019). Everyday self-defence: Hollaback narratives, habitus and resisting street harassment. The British Journal of Sociology, 70(5), 1709-1729. Doi: https://doi.org/10.1111/1468-4446.12699. 


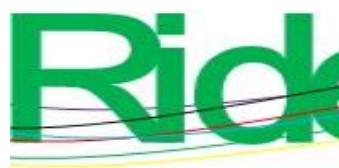

Revista Iberoamericana para la Investigación y el Desarrollo Educativo ISSN 2007-7467

González Moreno, M. J., Fernández Prados, J. S. and Cuenca-Piqueras, C. (2020). Fear for all women. Journal of Interpersonal Violence, 0886260520958390. Doi: https://doi.org/10.1177/0886260520958390.

Guerrera, F. (2018). \#Ni una más. El feminicidio en México, tema urgente en la agenda nacional. México: Aguilar.

Hawken S., Leao S. Z., Gudes, O., Izadpanahi, P., Viswanath, K. and Pettit C. (2020). Safer Cities for Women: Global and Local Innovations with Open Data and Civic Technology. In Hawken, S., Han, H. and Pettit, C. (eds.), Open Cities | Open Data. Palgrave Macmillan, Singapore. Doi: https://doi.org/10.1007/978-981-13-6605$5 \_4$.

Hebert, L. E., Bansal, S., Lee, S. Y., Yan, S., Akinola, M., Rhyne, M., Menendez, A. and Gilliam, M. (2020). Understanding young women's experiences of gender inequality in Lucknow, Uttar Pradesh through story circles. International Journal of Adolescence $\quad$ Youth, 25(1), 1-11. Doi: https://doi.org/10.1080/02673843.2019.1568888.

Hernández, D. and Gómez, J. C. (2021). Being a woman and studying laws: anthropological approaches to harassment and their resistance at a public university in Mexico City, Mexico. Cadernos de Saude Publica,37(2). Doi: https://doi.org/10.1590/0102-311X00024620

Hoor-Ul-Ain, S. (2020). Public sexual harassment mayhem on public transport in megacities-Karachi and London: A comparative review. Aggression and Violent Behavior, 52. Doi: https://doi.org/10.1016/j.avb.2020.101420

Infante-Vargas, D. and Boyer, K. (2021). Gender-based violence against women users of public transport in Saltillo, Coahuila, México. Journal of Gender Studies, 1-15. Doi: https://doi.org/10.1080/09589236.2021.1915753.

Lacey, A., Miller, R., Reeves, D. and Tankel, Y. (2021). From Gender Mainstreaming to Intersectionality: Advances in Achieving Inclusive and Safe Cities. In Public Space Reader (pp. 64-71). Routledge.

Lewis, S., Saukko, P. and Lumsden, K. (2021). Rhythms, sociabilities and transience of sexual harassment in transport: Mobilities perspectives of the London underground. Gender, $\quad$ Place \& Culture, 28(2), 277-298. Doi: https://doi.org/10.1080/0966369X.2020.1734540. 


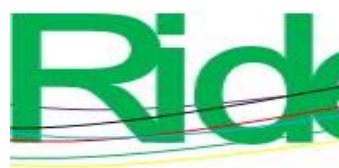

Revista Iberoamericana para la
Investigación y el Desarrollo Educativo
ISSN $2007-7467$

Logan, L. S. (2015). Street harassment: Current and promising avenues for researchers and activists. Sociology Compass, 9, 196-211. Doi: https://doi.org/10.1111/soc4.12248.

López, M. C. (2020). Estado del arte sobre el acoso sexual callejero: un estudio sobre aproximaciones teóricas y formas de resistencia frente a un tipo de violencia basada en género en América Latina desde el 2002 hasta el 2020. Ciencia Política, 15(30), 195-227.

Maffi, S., Malgieri, P. and Di Bartolo, C. (comps.) (2015). Smart choise for cities. Gender equality and mobility: mind the gap. CIVITAS Policy Note. Retrieved from https://civitas.eu/sites/default/files/civ_pol-an2_m_web.pdf

Medina, V. y Zapala, C. (2016). Representaciones sociales de las mujeres jóvenes sobre el acoso sexual callejero en la Ciudad de Puno. Punto Cero, 21(33), 60-81.

Mellgren, C., Andersson, M. and Ivert, A. K. (2018). "It happens all the time”: Women's experiences and normalization of sexual harassment in public space. Women \& Criminal Justice, 28(4), 262-281. Doi: https://doi.org/10.1080/08974454.2017.1372328.

México Evalúa (8 de marzo de 2021). En 2020, el 98.6\% de los casos de violencia sexual no se denunciaron. Recuperado de https://www.mexicoevalua.org/en-2020-el-98-6de-los-casos-de-violencia-sexual-no-se-denunciaron/

Mohamed, A. A. and Stanek, D. (2019). Street networks, pedestrian movement patterns and sexual harassment. Journal of Gender-Based Violence, 3(1), 7-28.

ONU Mujeres (2020). Iniciativa insignia mundial, ciudades seguras y espacios públicos seguros para mujeres y las niñas. Segundo compendio internacional de prácticas. Sección eliminación de la violencia contra las mujeres. Estados Unidos. Recuperado de https://www.unwomen.org//media/headquarters/attachments/sections/library/publications/2020/safe-cities-andsafe-public-spaces-international-compendium-of-practices-02es.pdf?la=es\&vs=2744.

Pedersen, L. (2020). Moving bodies as moving targets: A feminist perspective on sexual violence in transit. Open Philosophy, 3(1), 369-388. Doi: https://doi.org/10.1515/opphil-2020-0134.

Platzer, M. (2016). The Right to a Safe City for Women and Girls. In Kury, H., Redo, S. and Shea, E. (eds.), Women and Children as Victims and Offenders: Background, 


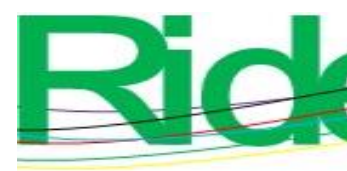

Revista Iberoamericana para la Investigación y el Desarrollo Educativo ISSN $2007-7467$

Prevention, Reintegration. Springer, Cham. Doi: https://doi.org/10.1007/978-3-31928424-8_25.

Quinones, L. M. (2020). Sexual harassment in public transport in Bogotá. Transportation Research Part A: Policy and Practice, 139, 54-69. Doi: https://doi.org/10.1016/j.tra.2020.06.018.

Royo, M. G., Parikh, P. and Belur, J. (2020). Using heat maps to identify areas prone to violence against women in the public sphere. Crime Science, 9(1), 1-15.

Sadler, N. (2019). Myths, masterplots and sexual harassment in Egypt. The Journal of North African Studies, 24(2), 247-270. Doi: https://doi.org/10.1080/13629387.2017.1419872.

Sen, A., Kaur, R. and Zabiliūte, E. (2020). Countering sexual violence in the Indian city. Gender, $\quad$ Place $\quad \& \quad$ Culture, 27(1), 1-12. Doi: https://doi.org/10.1080/0966369X.2019.1612856.

Talone, V. e Araujo, A. B. (2019). A evitação do assédio sexual no transporte público: uma leitura pragmatista das práticas de desconfiança de mulheres na cidade do Rio de Janeiro. Contemporânea-Revista de Sociologia da UFSCar, 9(3), 989-1008.

Toro, J. y Ochoa, M. (2017). Violencia de género y ciudad: cartografías feministas del temor y el miedo. Sociedad y Economía, (32), 65-84.

Torres Falcón, M. (2020). La interlocución del movimiento feminista con el gobierno mexicano: el caso de la alerta por violencia de género. Revista Mexicana de Estudios de los Movimientos Sociales, 4(2), 57-78.

Vallejo Rivera, E. y Rivarola, M. (2013). La violencia invisible: acoso sexual callejero en Lima metropolitana. Cuadernos de Investigación, (4), 1-19.

Vera-Gray, F. (2016). Men's stranger intrusions: Rethinking street harassment. Women's Studies International Forum, 58, 9-17. Doi: https://doi.org/10.1016/j.wsif.2016.04.001.

Walton, K. A. and Pedersen, C. L. (2021). Motivations Behind Catcalling: Exploring Men's Engagement in Street Harassment Behavior. Psychology \& Sexuality, (justaccepted). Doi: https://doi.org/10.1080/19419899.2021.1909648.

Wesselmann, E. D. and Kelly, J. R. (2010). Cat-calls and culpability: Investigating the frequency and functions of stranger harassment. Sex Roles, 63(7-8), 451-462. Doi: https://doi.org/10.1007/s11199-010-9830-2 